\title{
Controlled synthesis of noble metal nanomaterials: motivation, principles, and opportunities in nanocatalysis
}

\author{
RAFAEL S. GEONMONOND, ANDERSON G.M. DA SILVA and PEDRO H.C. CAMARGO \\ Instituto de Química, Departamento de Química Fundamental, Universidade de São Paulo, \\ Avenida Prof. Lineu Prestes, 748, 05508-000 São Paulo, SP, Brazil
}

Manuscript received on July 27, 2017; accepted for publication on September 6, 2017

\begin{abstract}
This review describes some principles of the controlled synthesis of metal nanoparticles, focusing on how the fundamental understanding of their synthesis in the solution-phase can be put to tailor size, shape, composition, and architecture. The maneuvering over these parameters not only enable the tuning of properties, but also the maximization and optimization of performances for various applications. Herein, we start with a brief description of metallic nanoparticles, highlighting the motivation for achieving physicochemical control in their synthesis. After that, we turn our attention to some important definitions and classifications as well as their unique properties such as surface and quantum effects. Moreover, we discuss the strategies for the controlled synthesis of metal nanomaterials based on the top-down and bottom-up approaches, focusing our discussion on their formation mechanisms in liquid-phase in terms of both thermodynamic and kinetic control. Finally, we point out the promising applications of controlled nanomaterials in the field of nanocatalysis and plasmon-enhanced catalysis, describing some of the current challenges in these fields.
\end{abstract}

Key words: controlled synthesis, noble-metals, nanomaterials, catalysis.

\section{INTRODUCTION}

Among the several classes of materials, noble metals such as silver $(\mathrm{Ag})$ and gold $(\mathrm{Au})$ have fascinated humanity for centuries since their discovery (Eustis and El-Sayed 2006). They used to be widely employed in many civilizations and religions as curative substances, bracelets, necklaces, ornaments, and decorative or sacred items (Vajtai 2013, Xiong et al. 2014). Although

\footnotetext{
Correspondence to: Pedro Henrique Cury Camargo

E-mail: camargo@iq.usp.br

* Contribution to the centenary of the Brazilian Academy of Sciences.
}

noble metals were primarily appreciated in their bulk form as a result of their beautiful and bright colors, their applications have recently become much more technological. This occurred due to recent progress on their fundamental physics and chemistry as well as advances in materials science for manipulating matter at the nanoscale (between $1-100 \mathrm{~nm}$ in size in at least one dimension) (Eustis and El-Sayed 2006, Vajtai 2013, Xiong et al. 2014). These attributes have led to the rise of the grand area encompassing metal nanomaterials. They differ from the corresponding bulk analogs in many ways, and this difference is the reason 
for the establishment of a field that describes their properties and performances (Vajtai 2013, Xiong et al. 2014).

In fact, metalnanomaterials haverevolutionized several applications in nanoscience. These include plasmonics, catalysis, sensing, electronics, photonics, information storage, medicine, energy conversion, cosmetics, among others (Arico et al. 2005, Lee and El-Sayed 2006, Polshettiwar and Varma 2010, Jariwala et al. 2013, Baffou and Quidant 2014, Kogure and Hama 2015, Pang et al. 2016). Their high and tunable performances arise from the fact that properties in metal nanomaterials strong correlate to their individual or combined physical and chemical features. For instance, properties are strongly dependent on composition (mono versus bimetallic), size (the effect of quantum confinement), geometric/shape (faceting or arrangement of atoms on the surface), and structure (hollow vs solid interiors). During the solution-phase synthesis of metal nanomaterials, all these parameters can be controlled so that, at least in principle, it is possible to optimize performances for a wealth of applications (Xia et al. 2009). As a classical example, controlling the shape of a metal nanoparticle provides an effective strategy to control their catalytic properties (Jin et al. 2012).

In addition to catalytic properties, tuning the size and shape of a metal nanoparticle provides an effective approach to control the collective oscillation of free electrons in a metal nanomaterial, which is referred as surface plasmon resonance (SPR) (Eustis and El-Sayed 2006). The SPR phenomenon is responsible for the beautiful and intense colors of $\mathrm{Au}$ nanoparticles having different sizes as shown in Figure 1 (Eustis and El-Sayed 2006, da Silva et al. 2014). The SPR excitation occurs as a result of the collective oscillation of the electrons in the metal in response to an oscillating electric field component from an incoming electromagnetic wave (Klar et al. 1998, Willets and Van Duyne 2007, Lu et al. 2009). This takes place
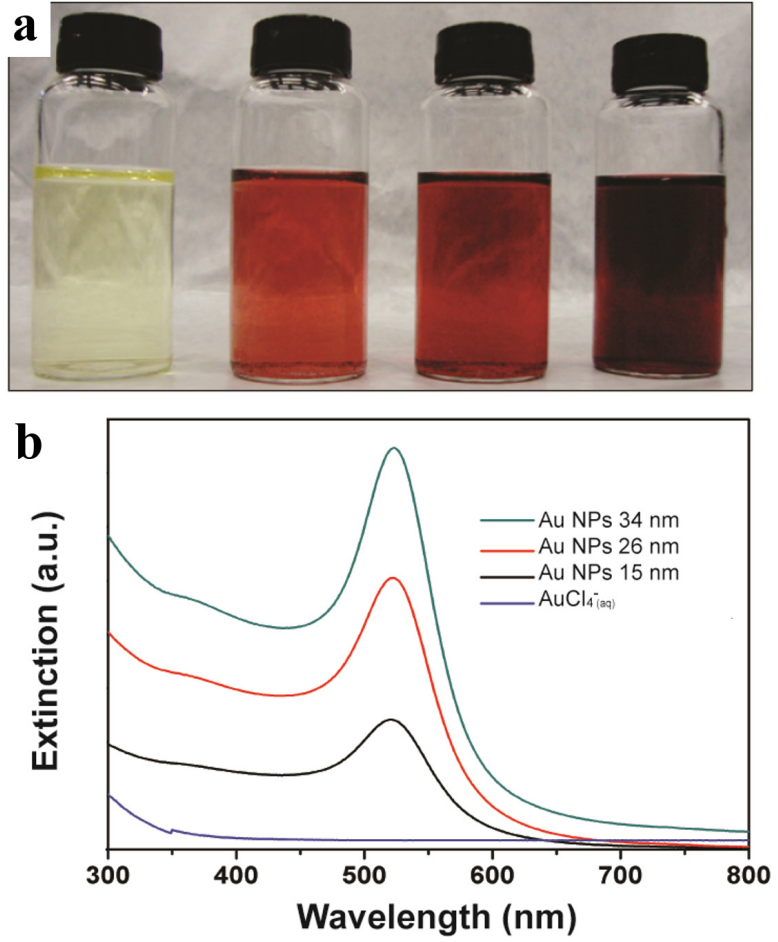

Figure 1 - a) Digital photographs of the $\mathrm{AuCl}_{4 \text { (aq) }}^{-}$solution and aqueous suspensions containing $\mathrm{Au}$ NPs $15 \pm 2.2,26 \pm$ 2.4 , and $34 \pm 3.0 \mathrm{~nm}$ in diameter (shown from left to right, respectively). b) UV-VIS extinction spectra recorded for the $\mathrm{AuCl}_{4(\mathrm{aq})}^{-}$solution and aqueous suspensions containing Au NPs $15 \pm 2.2,26 \pm 2.4$, and $34 \pm 3.0 \mathrm{~nm}$ in diameter (blue, black, red, and green traces, respectively). Adapted with permission from ref. (da Silva et al. 2014) Copyright 2014 Scielo Brazil.

in the visible or near-infrared range of the spectrum for metals such as $\mathrm{Cu}, \mathrm{Ag}$, $\mathrm{Au}$ (Klar et al. 1998, Willets and Van Duyne 2007, Lu et al. 2009). This unique light-matter interaction gives rise to several effects, including absorption and scattering of the incoming electromagnetic wave, the generation of intense local electric fields close to the surface of a metal nanoparticles, the formation of hot electrons and holes that can be used to enhance or drive chemical transformations, and localized heating as a result of plasmon decay (Kale et al. 2014, Linic et al. 2015). These unique optical properties have enabled applications in many areas that encompass surface-enhanced Raman scattering (SERS) detection, photothermal therapy, imaging, plasmonic catalysis, plasmonic solar cells, and 
artificial photosynthesis, for example (Jain et al. 2007, Catchpole and Polman 2008, Stiles et al. 2008, Baffou and Quidant 2014, Pang et al. 2016).

Due to these potential for applications in these fascinating areas as well as the possibility of improving or tailoring performances, metal nanoparticles in a variety of sizes, shapes (spheres, cubes, octahedra, rods, wires, plates, bipyramid, etc), compositions (Au, Ag, Pd, Pt, etc), and architectures (alloys, core-shell, rattles, and dendrites) have been pursued (Sun and Xia 2002, Xia et al. 2009, 2017, Niu et al. 2013). However, the level of synthetic control in terms of straightforwardness and robustness still remains challenging and restricted to a few systems. Traditionally, progress in this area has taken place by an observation-driven and trial and error approach (Corma 2016, da Silva et al. 2017, Xia et al. 2017). In this context, a transition to a design-driven methodology to the synthesis of nanomaterials with desired features for a target application represents the next chapter in the field of nanomaterials synthesis. This, in fact, will be imperative to enable massive impacts in industry, economy, and environment. In order to move towards a design-driven era in the synthesis of metal nanomaterials, it is central to fundamentally understand the steps involved in particle nucleation and growth (Viswanatha and Sarma 2007, You and Fang 2016). In this review, we will focus on the fundamental principles involved in the solutionphase synthesis of metal nanoparticles, emphasizing definitions and motivations, the theory of classical nucleation and growth, and some established strategies for controlling their sizes, shapes, and compositions (Xia et al. 2009, Camargo et al. 2015, You and Fang 2016). Finally, as an example, we will discuss the unique catalytic and optical applications of noble metal nanoparticles, highlighting recent progress in this field.

\section{METALLIC NANOMATERIALS: DEFINITIONS, CLASSIFICATIONS, AND MOTIVATIONS FOR CONTROLLED SYNTHESIS}

The term nanotechnology has been employed to describe the design, characterization, production, and application of nanomaterials (Vajtai 2013, Xiong et al. 2014). Nanomaterials have emerged as one of the most exciting areas of modern science and technology. Their development has significantly contributed to many applications ranging from catalysis to electronics and information storage. The rise of this area has also served as the driving force for the rapid development of metallic nanomaterials. Currently, research in metal nanomaterials can be considered as a multidisciplinary area which establishes a bridge between atoms and bulk solids by manipulating and generating metal particles at the nanoscale. It encompasses chemistry, physics, materials science, medicine, and biology.

As above mentioned, nanomaterials exhibit many remarkable chemical and physical properties that are different from both individual metal atoms and bulk counterparts. However, why are materials on this 1-100 nm range distinguished relative to those bulk counterparts? When multiple atoms are put together, the acquire properties that significantly differ relative those single atoms for obvious reasons. Firstly, by increasing the number of atoms in the particle, a perturbation of the atomic energy levels arises, leading to the formation of energy bands (Vajtai 2013, Xiong et al. 2014). Here, the energy difference between neighboring states is $\mathrm{E} / 2 \mathrm{~N}$ ( $\mathrm{E}$ is the width of the energy band and $\mathrm{N}$ is the number of atoms). Also, smaller sizes lead to higher surface to volume ratios. For example, Table I shows the relationships between particle size versus the number of surface atoms, shells, and percentage of surface atoms for a metal nanoparticle (Xia et al. 2009, Niu and Lu 2015). It can be observed that a dramatic increase in the percentage of surface atoms can be obtained as the 
TABLE I

Atoms percentage (\%) at a metal nanoparticle surface as a function of the total number of atoms and shells (Xia et al. 2009, Niu and Lu 2015).

\begin{tabular}{cccc}
\hline $\begin{array}{c}\text { Full-shell } \\
\text { clusters }\end{array}$ & Total number of atoms & Number of surface atoms & Surface atoms (\%) \\
\hline 1 shell & 13 & 12 & 92 \\
2 shells & 55 & 42 & 76 \\
3 shells & 147 & 92 & 63 \\
4 shells & 309 & 162 & 52 \\
5 shells & 561 & 252 & 45 \\
6 shells & 923 & 362 & 39 \\
\hline
\end{tabular}

size of nanoparticles decreases. The large surface to volume ratio of metallic nanostructures is the origin of a number of unique properties, especially chemical reactivity which serves as the basis for catalysis (Albonetti et al. 2015).

Besides their sizes (the effect of quantum confinement, as well as the proportions of atoms at corners, edges, and faces), it has been well-established that the properties of metal nanoparticles are strongly dependent on composition (mono- versus bi- or multimetallic, and the spatial distributions of different elements), shape (faceting or arrangement of atoms on the surface), and structure (convex, concave, solid, hollow, or porous) (Xia et al. 2009). A precise control only over the shape of a metal nanoparticle can be employed as a simple and versatile strategy for tailoring particle performance for a targeted application. For example, it has been demonstrated that Pd nanocubes bound by $\{100\}$ facets are significantly more active relative to the other shapes toward the formic acid oxidation reaction as shown in Figure 2 (Jin et al. 2012). As $\{100\}$ surface facets are more active relative to $\{111\}$ for the electro-oxidation of formic acid, a gradual increase in the current densities was observed as the shape was changed from cubes to truncated cubes, cuboctahedrons, truncated octahedrons, and finally octahedrons, as these shapes gradually enable an increased exposure of $\{111\}$ relative to $\{100\}$ surface facets (Jin et al. 2012). These results can

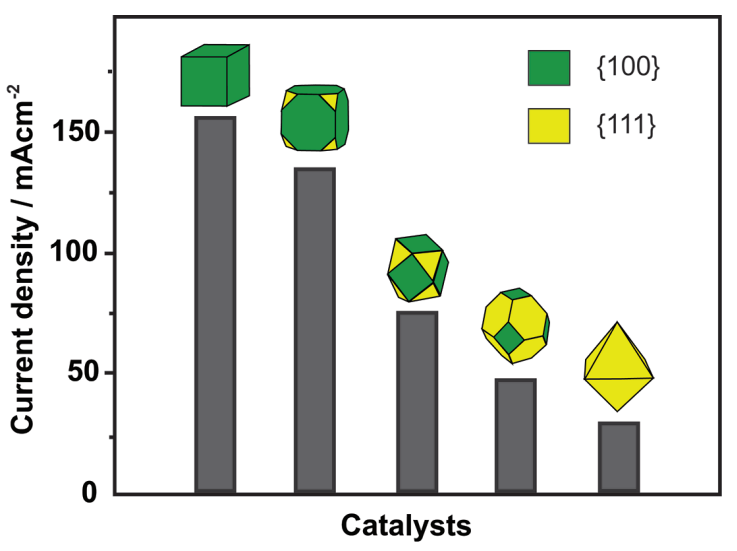

Figure 2 - Maximum current densities for formic acid oxidation employing Pd nanocrystals displaying controlled shapes as electrocatalysts. Adapted with permission from ref. (Jin et al. 2012) Copyright 2012 Royal Society of Chemistry.

be explained as a function of the differences in the surface energy of each crystal facet. Nanocrystals displaying $\{100\}$ surface facets can interact more efficiently with the formic acid molecule, leading to a higher catalytic activity (Xia et al. 2009, Jin et al. 2012). It is noteworthy that surface energy $(\gamma)$ can be defined as the energy required for creating a unit area of "new" surface, or as the excess free energy per unit area for a particular crystallographic face, according to Equation 1 (Xia et al. 2009, Xiong et al. 2014):

$\gamma=(1 / 2) \mathrm{N}_{\mathrm{b}} \varepsilon \rho$

where $\mathrm{N}_{b}$ is the number of bonds that need to be broken to produce the new surface, $\varepsilon$ is the bond strength, and $\rho$ is the density of surface atoms. For 
an $f c c$ structure (metal such as $\mathrm{Ag}, \mathrm{Au}, \mathrm{Pd}, \mathrm{Pt}, \mathrm{Cu}$ ) with a lattice constant of $a$, the surface energies of the low-index crystallographic facets that typically encase noble metal-nanocrystals can be estimated as $\gamma 100=4(\varepsilon / \mathrm{a} 2), \gamma 110=4.24(\varepsilon / \mathrm{a} 2)$, and $\gamma 111=$ 3.36(ع/a2), (Xia et al. 2009, Xiong et al. 2014) resulting in the energetic sequence of $\gamma 111<\gamma 100<$ $\gamma 110$ as shown in Figure 3.

Since the Faraday's synthesis of colloidal Au nanoparticles, (Faraday 1857) researchers have tried to establish a reasonable way to classify nanostructures. One of the most accepted definitions categorizes a different kind of nanomaterials according to their sizes in three dimensions (Vajtai 2013, Niu and Lu 2015). They can be classified into four groups: i) zero-dimensional (0D); i) onedimensional (1D); iii) two-dimensional (2D); and iv) three-dimensional (3D) (Vajtai 2013, Niu and Lu 2015). 0D nanomaterials can be defined as having nanoscale features along $\mathrm{x}, \mathrm{y}$, and $\mathrm{z}$ directions. 1D and 2D nanostructures are anisotropic materials having more complex structures with different growth rates in specific directions. For example, nanowires, nanorods, and nanotubes can be classified as $1 \mathrm{D}$, while nanosheets, nanoplates, and nanowalls can be classified as 2D. Finally, 3D nanomaterials could be produced by a combination of $0 \mathrm{D}, 1 \mathrm{D}$, and 2D nanostructures to produce superlattices and microcrystals.

\section{APPROACHES FOR THE CONTROLLED SYNTHESIS OF METAL NANOMATERIALS}

Essentially, there are two main basic approaches employed in the synthesis of metal nanoparticles as shown Figure 4. The first is the top-down approach (Walt 2002, Biswas et al. 2012). Here, sophisticated macroscopic materials and expensive techniques are employed to successively decrease the dimensions of a bulk material or incorporate nanoscale features into them. These include photolithography, e-beam lithography, dip-pen lithography, molecular beam

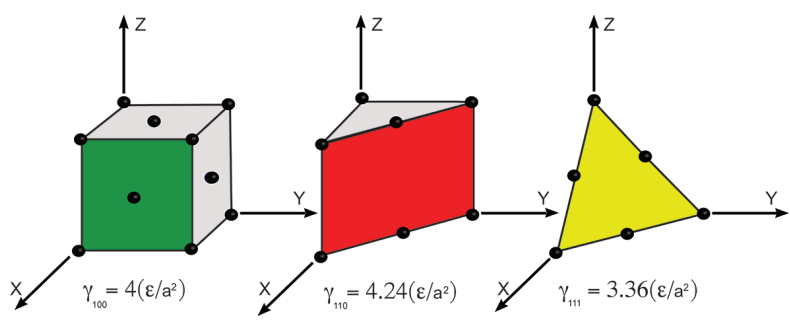

Figure 3 - Atomic arrangements and surface energy values for $\{100\},\{110\}$, and $\{111\}$ surface facets (from left to right, respectively).

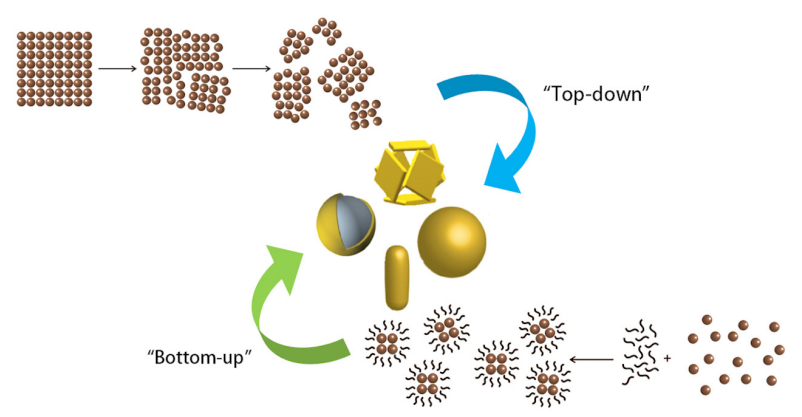

Figure 4 - Top-down and bottom-up approaches for producing controlled nanomaterials.

epitaxy, among others (Walt 2002, Biswas et al. 2012). The second method relies on bottom-up, that implies that the nanostructures are obtained from atoms as the building blocks. This includes chemical solution-phase synthesis, vapor deposition (CVD), and plasma/flame spraying synthesis (Walt 2002, Biswas et al. 2012). Bottom-up approaches have gained enormous attention because they are generally less expensive, can be controlled by manipulating many experimental parameters, and present the capability to reach industrial scale (e.g., solution-phase reactions) (Walt 2002, Biswas et al. 2012). However, the control over the uniformity of the produced nanomaterials is still challenging.

Among different methods for the controlled synthesis of metal nanomaterials, solution-phase approaches offer particular advantages that include the facile stabilization by the addition of proper capping and stabilizing agents, easy extraction/separation from the reaction mixture (by centrifugation, for example), straightforward 
surface modification/functionalization, potential for large-scale production, and versatility regarding the several experimental parameters that can be controlled during the synthesis (temperature, nature of precursors, stabilizers, solvent, reducing agents, their molar ratios, concentrations, etc) (Xia et al. 2009). Surprisingly, substantial developments on the solution-phase and controlled synthesis of metallic nanomaterials with a variety of shapes and sizes were achieved only recently. Figure 5 illustrates a variety of shapes that have been described (Xia et al. 2009, Camargo et al. 2015). Despite this progress, a detailed atomic understanding of the mechanisms of nucleation and growth stages during nanoparticle formation is still not completely understood, and most fundamentals are currently borrowed from classical colloidal theories as discussed below.

\section{FORMATION OF A METAL NANOPARTICLE: THE MECHANISM FOR NUCLEATION AND GROWTH}

In the context of colloidal synthesis, nucleation represents the very first step that atomic or molecular species must undertake before emerging as noble-metal nanocrystals (Leite and Ribeiro 2012, Thanh et al. 2014, Camargo et al. 2015). In a one-pot synthesis of metal nanocrystals, a precursor is reduced or decomposed to generate atoms, that are the building blocks (also referred to as monomers) of a nanoparticle. In general, the atoms can take two distinct routes: homogeneous nucleation (or self-nucleation) to generate nuclei/ seeds and heterogeneous nucleation, a process involving the deposition of atoms on the surface of existing seeds or surfaces (Leite and Ribeiro 2012, Thanh et al. 2014, Camargo et al. 2015).

In the case of homogeneous nucleation, much of the understanding is based on the assertions first put forward by LaMer and Dinegar (LaMer and Dinegar 1950). These studies date back from the 1950s and are still the basis of our understanding of the synthesis of uniform nanomaterials (including metallic, polymeric, semiconducting, and oxide systems) (You and Fang 2016). In this case, the control of the morphology of colloidal metal nanocrystals has been mainly understood by adopting atom-mediated nucleation and growth theories, in which atoms are the basic building blocks for the nucleation and growth. Nucleation can be defined as the process by which building blocks (metal atoms in the synthesis of metal nanomaterials) arrange themselves according to their crystalline structure to form a site upon which additional building blocks can deposit over and undergo subsequent growth (Leite and Ribeiro 2012, Thanh et al. 2014, Camargo et al. 2015). In this context, nuclei correspond to infinitesimal clusters consisting of very few atoms of the growth species. Such nucleation and growth processes can, therefore, be described by referring to the evolution of the atomic concentration over time, as illustrated by the so called LaMer curve depicted in Figure 6 . The nucleation and growth process of colloidal metal nanocrystals described by the LaMer curve can be divided into three main stages: i) atom production, ii) nucleation from atoms aggregation and iii) nanocrystal growth from the atomic addition (You and Fang 2016).

From these studies, the concept of "burst nucleation" has been established to be crucial for the synthesis of monodispersed particles. Burst nucleation refers to the formation of a large number of nuclei in a short period of time, followed by growth without additional nucleation (Park et al. 2007). This allows nuclei to have similar growth histories and, therefore, yield nanoparticles with same sizes. This concept is normally referred to as the separation of nucleation and growth stages during the synthesis. For instance, if nucleation and growth could occur simultaneously, the nuclei growth histories would be significantly different and heterogeneous size distributions would be obtained. 


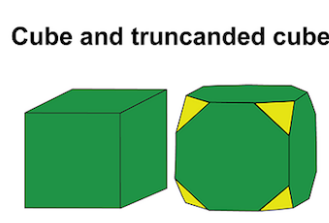

Pd, Ag, Au, Pt, Cu, Rh, Bi, Fe

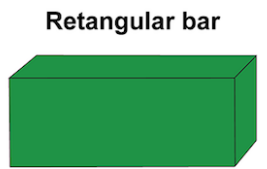

$\mathrm{Pd}, \mathrm{Ag}, \mathrm{Pt}$

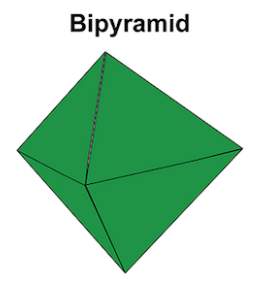

$\mathrm{Pd}, \mathrm{Ag}$

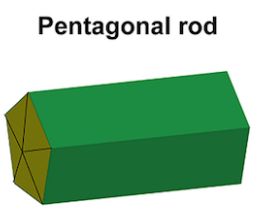

$\mathrm{Pd}, \mathrm{Ag}, \mathrm{Au}, \mathrm{Cu}$

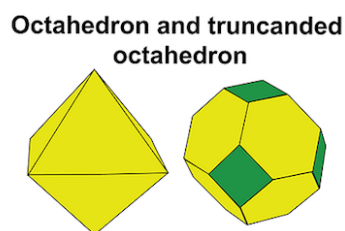

$\mathrm{Pd}, \mathrm{Ag}, \mathrm{Au}, \mathrm{Pt}$

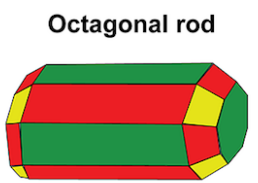

$\mathrm{Pd}, \mathrm{Au}, \mathrm{Fe}, \mathrm{Co}, \mathrm{Ni}$

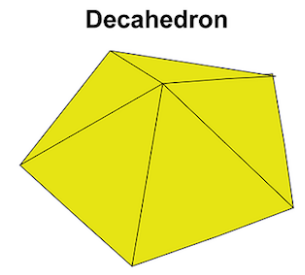

$\mathrm{Pd}, \mathrm{Ag}, \mathrm{Au}$

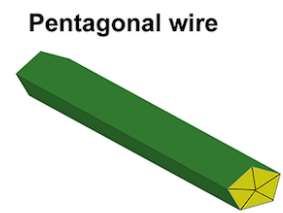

$\mathrm{Ag}, \mathrm{Au}, \mathrm{Cu}$

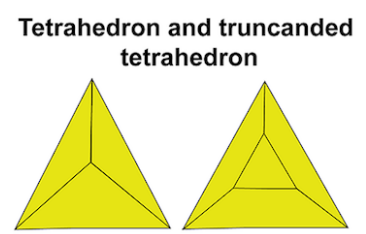

$\mathrm{Ag}, \mathrm{Au}, \mathrm{Pt}, \mathrm{Rh}$

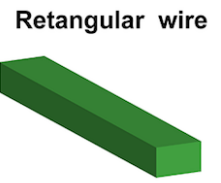

$\mathrm{Pb}, \mathrm{In}, \mathrm{Sn}, \mathrm{Sb}, \mathrm{Fe}, \mathrm{Co}$

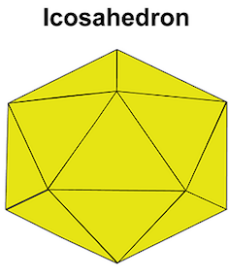

$\mathrm{Pd}, \mathrm{Au}$

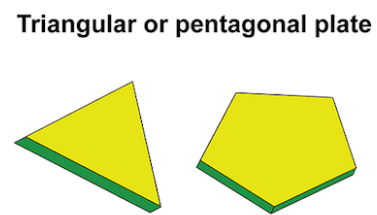

Pd, Ag, Au, Cu, Pd, Bi, Co, Ni

$$
\{100\} \square\{111\} \square\{110\}
$$

Figure 5 - Different shapes that have been enabled for a range of metals nanocrystals by solution- phase synthesis.

Figure 6 depicts the Lamer plot, which shows the change in the atomic concentration of the solute (growth species) during the nucleation and growth processes as a function of time. This plot is also very useful for illustrating the concept of burst nucleation. In the first stage of the Lamer curve, metallic atoms are produced either through a reduction of metallic ions or the thermal decomposition of organometallic compounds (Leite and Ribeiro 2012, Thanh et al. 2014, Camargo et al. 2015). Once the atomic concentration exceeds the point of supersaturation (Cnu min), in the second stage, atoms start to aggregate to form stable small clusters (i.e., nuclei) via self- (or homogeneous) nucleation (Leite and Ribeiro 2012, Thanh et al. 2014, Camargo et al. 2015). Afterward, the concentration of atoms quickly drops below the minimum supersaturation level (Cnu min) and no additional nucleation events will occur. Then, in the third stage, the size of the nuclei gradually increases due to the continuous addition of metal atoms. In this stage, once a cluster has grown 


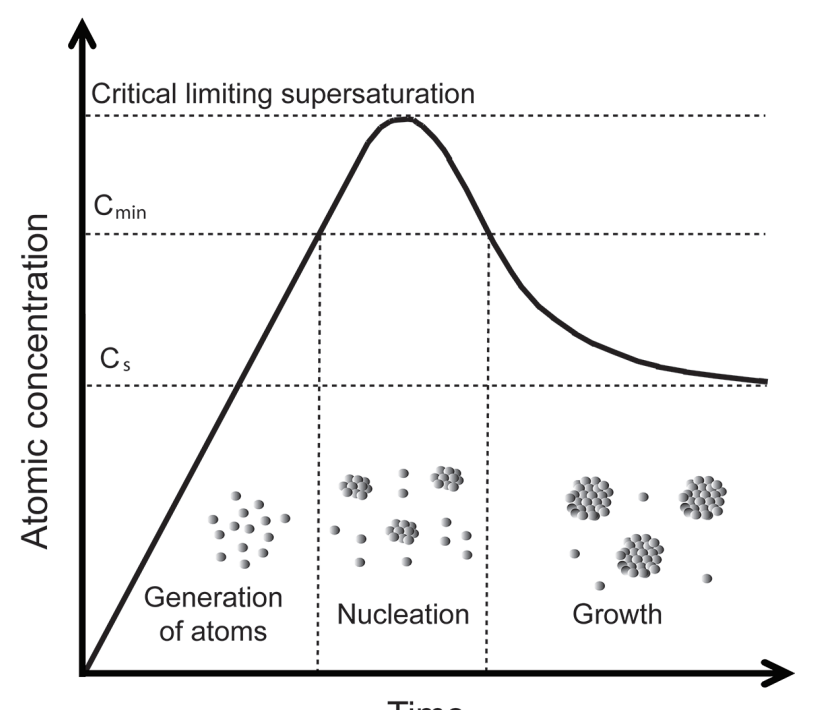

Time

Figure 6 - Variations in the atomic concentration of growth species in solution as a function of time during the generation of atoms, nucleation, and growth stages. Adapted with permission from ref (Xia et al. 2009) Copyright 2009 Wiley$\mathrm{VCH}$.

past a certain critical size, the activation energy for structural fluctuation will become so high that the cluster eventually becomes locked into a welldefined structure. This critical point marks the birth of a seed. This seed further grows to form the final nanocrystal through the addition of metal atoms until the concentration decreases to Cs (solubility concentration of nanocrystals) (Leite and Ribeiro 2012, Thanh et al. 2014, Camargo et al. 2015).

However, the classical theory does not explain the various crystallization pathways and outcomes that emanate from the reaction mixture when the shape-controlled synthesis of noblemetal nanoparticles is concerned. Specifically, the classical nucleation theory included a set of oversimplified assumptions, such as i) the nucleus is modeled as a perfect sphere, ii) the density and surface energy of the nucleus is size-invariant, and iii) the nucleus grows by taking one monomer at a time (Xia et al. 2017). Since nucleation is a highly dynamic process that involves irregularly shaped nuclei that grow with size-dependent surface energies, internal defects, impurities, and nucleusnucleus collisions, a more comprehensive theory is necessary to properly model the nucleation process.

As nuclei correspond to infinitesimal clusters consisting of very few atoms formed as the new solid phase in solution, structure fluctuations may occur in the nuclei with the incorporation of defects to minimize surface energy until they evolve into seeds (Gilroy et al. 2017). The seeds can be defined as species that are larger than the nuclei that are locked into a specific structure, in which structure fluctuation does not occur. This is because they become energetically costlier as the size increases. Interestingly, for noble-metals that include $\mathrm{Ag}, \mathrm{Au}$, $\mathrm{Pd}$, and Pt, it has been established that the final shape assumed by the nanoparticle is determined by both the structure of its corresponding seed and the relative binding affinities of capping agents to its distinct surface facets. Considering their $f c c$ structure, seeds can assume single-crystalline, single-twinned, multiple-twinned and/or stacking faults structures (Gilroy et al. 2017). Thus, the key for the synthesis of metal nanocrystals with controlled shapes relies on maneuvering nucleation and growth processes so that only one kind of seed, with the proper structure, is present in the reaction mixture. Figure 7 illustrates the typical seeds available to an $f c c$ metal (Ag, $\mathrm{Au}, \mathrm{Pd}$, and $\mathrm{Pt}$ ) and how the structure of the seeds play an important role in determining the final shape of a metal nanocrystal in solution-phase synthesis (Gilroy et al. 2017).

The structure of seeds during solution-phase synthesis (single crystalline, single-twinned, or multiple-twinned) are dependent upon many parameters that include thermodynamic and kinetic considerations, while the relative population distribution of seeds can be further influenced by oxidative etching processes (Gilroy et al. 2017). When the synthesis in under thermodynamic control, the population of seeds displaying different structures will be determined by the statistical 


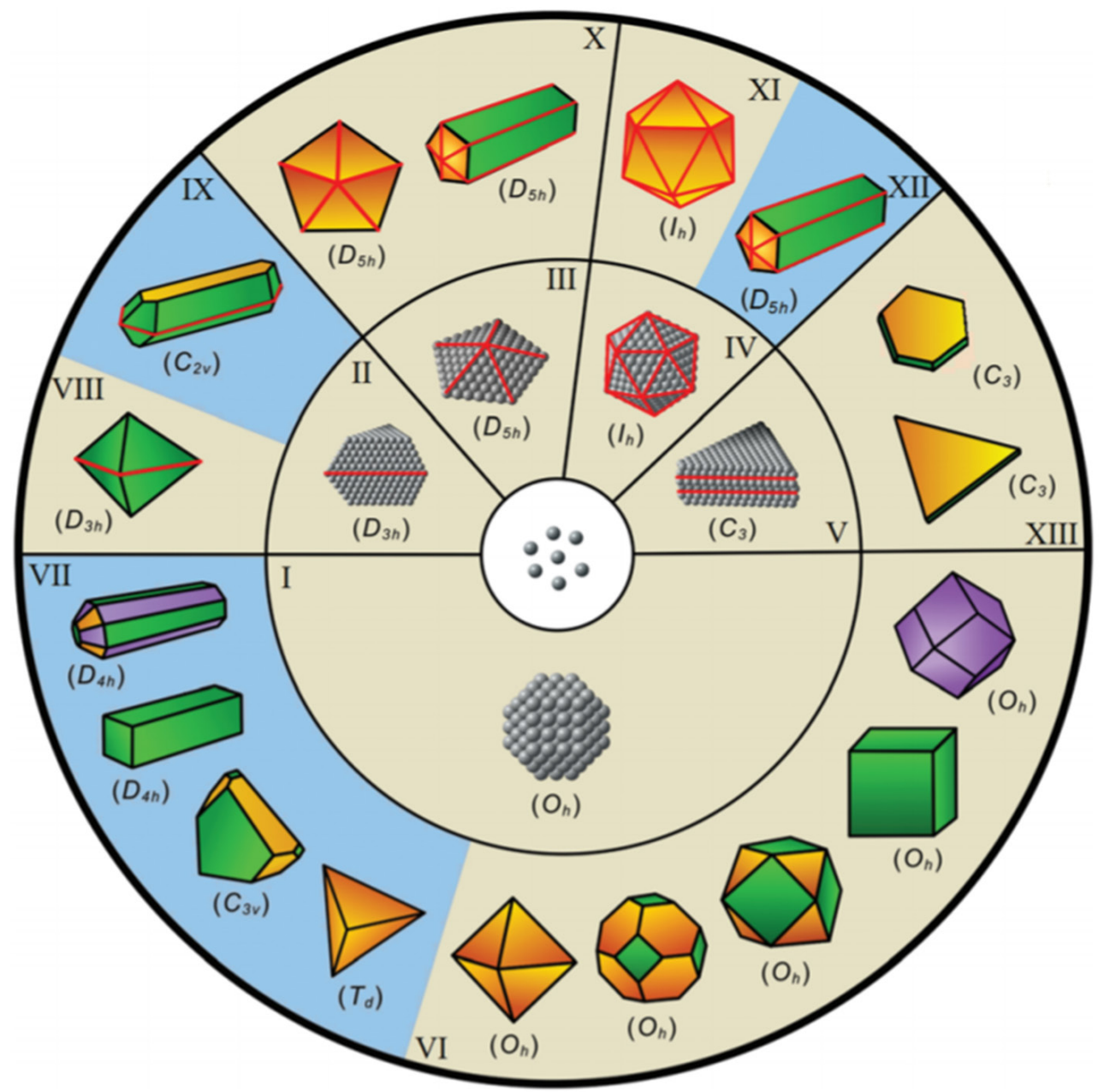

Figure 7 - Main stages of controlled synthesis of metal nanomaterials with face-centered cubic $(f c c)$ structure: (i) nucleation (in the center); (ii) growth of nuclei into seeds (in the middle ring); and (iii) evolution of the seeds into the final nanocrystal (in the outer ring). The shape assumed by the nanocrystal depends on the structure of its corresponding seed and the relative binding affinities of capping agents to its distinct surface facets. The red lines indicate the twin defects or stacking faults in a seed or nanocrystal while the yellow, green and purple colors correspond to the $\{111\},\{100\}$, and $\{110\}$ facets, respectively. When there is a change in the background color, symmetry breaking is involved during the growth. Adapted with permission from ref (Gilroy et al. 2017) Copyright 2017 Royal Society of Chemistry.

thermodynamics of their free energies. The most stable (lowest energy) seeds will be favored, and surface energy considerations are crucial in understanding and predicting the structure of the produced seeds (Gilroy et al. 2017). For example, Figure $8 \mathrm{a}$ shows a typical phase diagram for $\mathrm{Au}$ seeds as functions of particle size and temperature, indicating that multiply twinned and single-crystal seeds are favored at relatively small and large sizes.
Therefore, by varying the temperature and fixing a specific particle size, it is possible to control colloidal noble-metal nanocrystals displaying controlled sizes and shapes.

On the other hand, kinetic control relies on the manipulation of the structure/population of seeds containing different numbers of twin defects by decreasing the reduction or decomposition rate of a precursor that is responsible for providing metal 
atoms (growth species) (Gilroy et al. 2017). It has recently discovered that the initial reduction rate of a precursor is crucial to the formation of seeds with a particular internal structure in high purity. Specifically, the initial reduction rates necessary for generating different types of seeds tend to decrease in the order of single-crystal, singly twinned, multiply twinned, and stackingfault-lined seeds (Gilroy et al. 2017). Figure 8b shows the percentages of $\mathrm{Pd}$ seeds with different internal structures as a function of the initial rate for the reduction of $\mathrm{PdCl} 42-$. Interestingly, the existence of different ranges of reduction rates can be observed, within which the purity of seeds could approach $100 \%$. The reduction rate could be experimentally adjusted by temperature, as well as the type and concentration of reducing agent and precursor involved in the synthesis.

Over the last decade, a series of theories have been developed to describe the nucleation and growth of colloidal noble-metal nanocrystals displaying controlled sizes and shapes (Wiley et al. 2007, Xiong and Xia 2007, Xia et al. 2009, 2017, Niu et al. 2013). We have mentioned that the solution-phase synthesis of metal nanomaterials is comprised of nucleation and growth stages. In the shape-controlled synthesis of metal nanomaterials, these stages can be further subdivided into nucleation, the growth of nuclei into seeds, and growth and evolution of the seeds into the final nanocrystal. As a means to avoid the enigmatic processes that are involved in homogeneous nucleation, monomers or even the precursors can be added to preformed nanocrystals (the so-called seeds) at concentrations below the supersaturation level. During this process, monomers undergo phase transformation directly on the surface of the employed seed, the process so-called heterogeneous nucleation (Granasy et al. 2014). This approach enables the efficient separation of nucleation and growth processes and thus the synthesis of a variety of sophisticated nanomaterials, which may or may
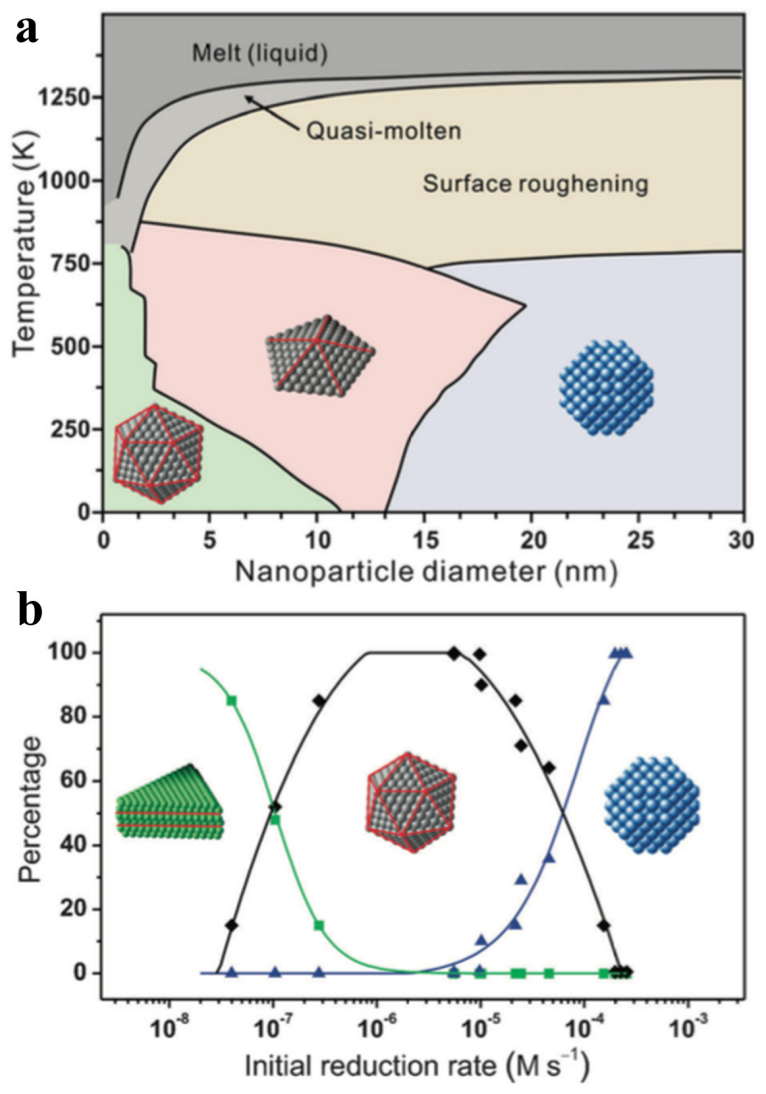

Figure 8 - Thermodynamic and kinetic approaches to controlling the internal structure of seeds. a) Phase diagram of Au seeds as a function of temperature and nanoparticle size; b) Population of Pd nanocrystals as a function of the initial reduction rate, displaying plates with stacking faults (orange), multiply twinned icosahedra (purple), and singlecrystal cuboctahedra (blue). Adapted with permission from refs (Barnard et al. 2009, Wang et al. 2015) Copyright 2009 and 2015 American Chemical Society, respectively.

not replicate the morphological features of the nanoparticles employed as seeds (Xia et al. 2017). In fact, notable examples have been demonstrated for structures composed of $\mathrm{Ag}, \mathrm{Au}, \mathrm{Pd}, \mathrm{Pt}$, and $\mathrm{Cu}$ covered by low-index facets such as cubes, octahedra, cuboctahedra, bypyramid, rectangular bars, and pentagonal rods or wires (Xiong and Xia 2007, Liao et al. 2008, Chen et al. 2009, Cobley et al. 2009). Additionally, nanocrystals covered by highindex facets, including concave cubes or octahedra, octopods, Decahedron, icosahedron, and triangular/ pentagonal plates, have also been prepared with great uniformity (Xiong and Xia 2007, Liao et al. 
2008, Chen et al. 2009, Cobley et al. 2009, Xia et al. 2009). Figure 9 summarizes TEM and SEM images of a variety of shapes for $\mathrm{Ag}$ and $\mathrm{Au}$ nanomaterials that have been successfully reported by the solution phase synthesis (Nikoobakht and El-Sayed 2003, Wiley et al. 2007, Zhang et al. 2010, Chen et al. 2014, Lohse et al. 2014, Rodrigues et al. 2017).

\section{SYNTHESIS OF HOLLOW NANOMATERIALS: THE GALVANIC REPLACEMENT REACTION}

Among the variety of nanomaterial varieties developed in past decades, nanostructures displaying hollow interiors and porous and thin walls have attracted significant attention because of their special and unique properties such as high surface areas, high porosity, and high reactivity (as a consequence of high concentration of defective sites and ultrathin walls) (An and Hyeon 2009, Xia et al. 2013). Nanostructures with hollow interiors have been extensively studied for their applications in microencapsulation, catalysis, drug delivery, and protection of environmentally sensitive biological species (An and Hyeon 2009, da Silva et al. 2017). Hollow nanostructures based on metals are intriguing to synthesize and study because they may exhibit plasmonic and catalytic properties that are different from their solid counterparts. For example, Prevo and co-workers have demonstrated that the surface plasmon resonance (SPR) band of gold nanoshells could be smoothly tuned over the spectral regime from 600 to $900 \mathrm{~nm}$ by varying their diameter and shell thickness (Prevo et al. 2008). In comparison, the SPR band of solid, spherical colloids of gold can only be tuned around $520 \mathrm{~nm}$ by as much as $\sim 50 \mathrm{~nm}$ (Prevo et al. 2008).

Among several approaches that have been reported, methods based on the Ostwald ripening, Kirkendall effect, galvanic replacement, and oxidative etching have been successfully employed for fabricating hollow nanomaterials (Sun et al. 2003, Yin et al. 2004, da Silva et al. 2017).
The galvanic replacement reaction is especially interesting as it allows us to easily control the sizes, shapes, compositions and internal structures, leading to the formation of nanomaterials displaying a high surface to volume ratio and large pore volume (Xia et al. 2013, da Silva et al. 2017). More specifically, this strategy is promising due several factors: (i) galvanic replacement process between a metallic nanoparticle (sacrificial template) and another metal precursor (having a higher standard reduction potential) often occurs spontaneously; (ii) this procedure is quite versatile and can be adapted for almost all metal nanoparticles templates and salt precursors; and (iii) it represents a simple route to the synthesis controlled nanostructures with ultrathin walls, in only one step, in which the composition of the nanoparticles can be easily controlled by adjusting the sacrificial template/ metal precursor molar rations during the synthesis (Xia et al. 2013, da Silva et al. 2017).

A galvanic replacement reaction, extensively employed for the synthesis of hollow metal nanomaterials, can be described as an oxidationreduction (redox) process. In this case, a redox reaction occurs when electrons are transferred from a metal nanoparticle (a reducing agent or sacrificial template) to ions in solution of a second metal (an oxidizing agent) (Xia et al. 2013, da Silva et al. 2017). The difference in the reduction potentials of both metals employed in the galvanic replacement reaction acts as the driving force in this process, being the reduction potential of the metal precursor necessarily higher than metal sacrificial template at the reaction conditions. Taking the galvanic replacement reaction between Ag nanoparticles and $\mathrm{HAuCl} 4$ precursor as an example, the galvanic process can be understood as a sum of the two following half equations as shown below:

$$
\begin{array}{ll}
\mathrm{Ag}_{(\mathrm{aq})}^{+}+1 \mathrm{e}^{-} \rightarrow \mathrm{Ag}^{0} & \varepsilon_{1}^{0}=0.8 \mathrm{~V} \\
\mathrm{AuCl}_{4}^{-}(\mathrm{aq})+3 \mathrm{e}^{-} \rightarrow \mathrm{Au}^{0}+4 \mathrm{Cl}^{-(\mathrm{aq})} & \varepsilon_{2}{ }^{0}=1.0 \mathrm{~V}(
\end{array}
$$




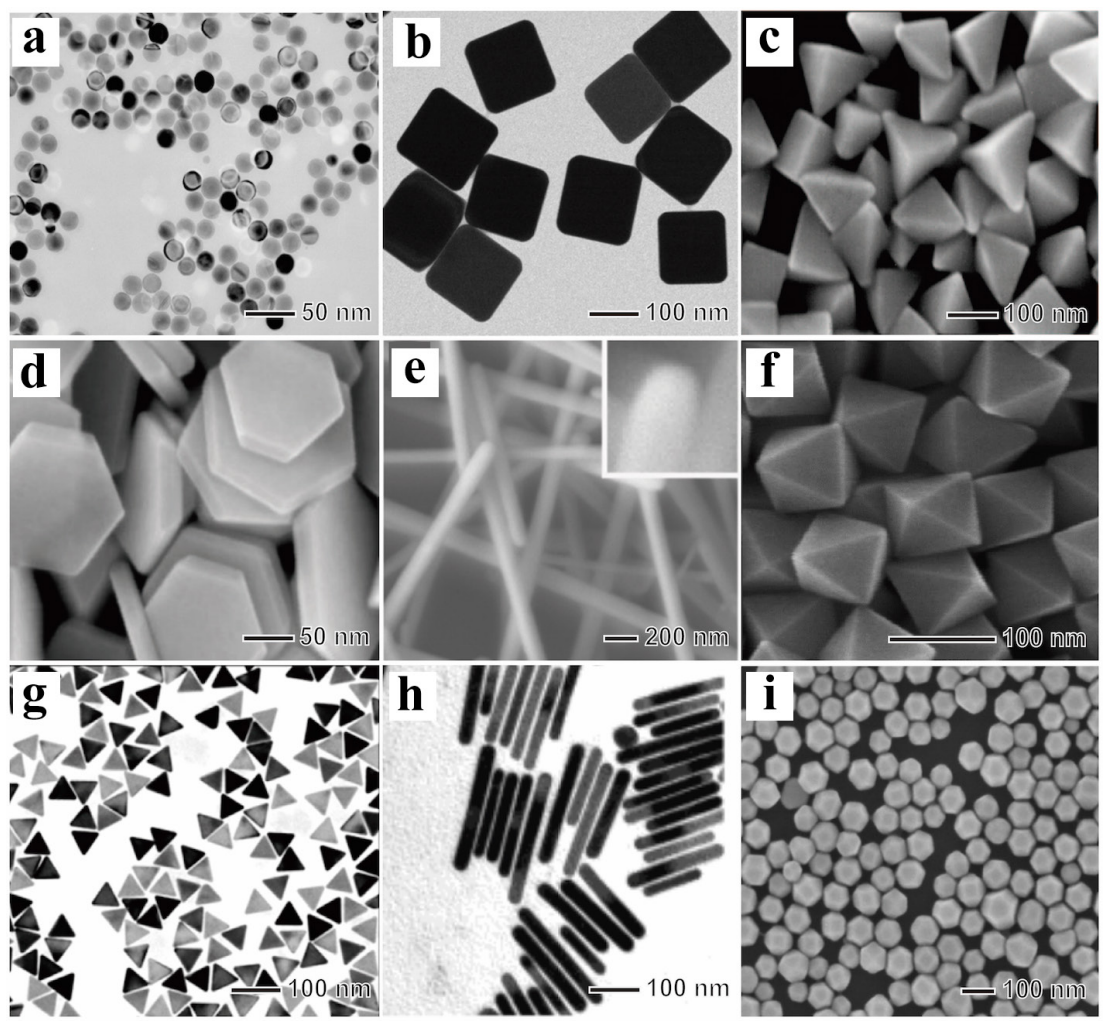

Figure 9 - a-i) SEM and TEM images for a variety of shapes that have been successfully reported by the solution phase synthesis. a) spheres; b) cubes; c) tetrahedrons; d) hexagonal plates; e) wires; f) octahedra; g) triangular plates; h) rods and, i) truncated cubes. Adapted with permission from: a, c and i) ref. (Wiley et al. 2007) Copyright 2007 American Chemical Society, b) ref. (Zhang et al. 2010) Copyright 2010 American Chemical Society; d) ref. (Wiley et al. 2007) Copyright 2007 American Chemical Society; e) ref.(Rodrigues et al. 2017) Copyright 2017 Scielo Brazil; f) ref. (Lohse et al. 2014) Copyright 2014 American Chemical Society; (G) ref. (Chen et al. 2014) Copyright 2014 American Chemical Society, and h) ref. (Nikoobakht and El-Sayed 2003) Copyright 2003 American Chemical Society.

And the final balanced equation:

$$
\begin{aligned}
& \mathrm{AuCl}^{-}{ }_{(\mathrm{aq})}+3 \mathrm{Ag}^{0} \rightarrow 3 \mathrm{Ag}^{+}{ }_{(\mathrm{aq})}+\mathrm{Au}^{0}+4 \mathrm{Cl}^{-}{ }_{(\mathrm{aq})} \quad \varepsilon_{3}{ }^{0}= \\
& 0.2 \mathrm{~V}
\end{aligned}
$$

The composition and structure of hollow nanomaterials obtained by the galvanic replacement reaction can be easily controlled by adjusting the molar ratio between $\mathrm{Ag}$ and $\mathrm{AuCl} 4-(\mathrm{aq})$ precursor added into the reaction mixture (Xia et al. 2013, da Silva et al. 2017). In this case, when a Ag nanosphere is kept in contact with small amounts of an aqueous AuCl4-(aq) solution, the Ag atoms from the sphere surface with high surface energy (defects, stacking faults, among others) can quickly be oxidized and dissolved by AuCl4-(aq) ions, producing Ag+ ions into the suspension reaction. This leads to the formation of small cavities at the nanoparticle surface as shown in Figure 10. At the same time, $\mathrm{Au}$ atoms are deposited at the nanoparticle surface because of the reduction of the AuCl4-(aq) ions precursor (Xia et al. 2013, da Silva et al. 2017). As $\mathrm{Au}$ and $\mathrm{Ag}$ have a great miscibility as a result of the similar face-centered cubic $(f c c)$ crystal structure and lattices constants that are close to each other $($ agold $=4.079 \AA ;$ asilver $=4.086 \AA)$, the initial 
$\mathrm{Au}$ atoms tend to deposit as a uniform thin $\mathrm{Au}$ layer at the Ag nanoparticle surface (Figure 13a, e). (Xia et al. 2013, da Silva et al. 2017). This the first step leads to the formation of an incomplete $\mathrm{Au}$ layer, which cannot prevent that intern layers from reacting with AuCl4-(aq) precursor. Subsequently, small cavities at the nanoparticle surface serves as the main sites for continuous galvanic replacement between Ag and AuCl4-(aq) precursor. As more $\mathrm{Ag}$ is replaced by Au by the increasing the amount of metal precursor into the reaction suspension, it is expected the formation of $\mathrm{AgAu}$ nanoshells displaying larger holes together with $\mathrm{Ag}-\mathrm{Au}$ alloying process leading to the formation of a homogenous distribution of $\mathrm{Ag}$ and $\mathrm{Au}$ at the nanoparticle surface (Figure 10) (Xia et al. 2013, da Silva et al. 2017). Finally, if this reaction is continued even further, the observed holes inside the nanostructure become large, leading to the rupture of the nanoshell morphology and resulting in the formation of several polydisperse Au nanoparticles (Figure 10). This simple approach can be extended for a variety of metal nanoparticles templates and salt precursors, representing a simple route to the synthesis controlled nanostructures with ultrathin walls, in one step, in which the composition can be easily controlled by the metallic precursor solutions employed and their concentrations (Xia et al. 2013, da Silva et al. 2017). For example, Figure 11 illustrates typical hollow nanomaterials produced by reacting silver nanospheres, nanocubes, triangular nanoplates, and nanowires with PdCl42(aq) or AuCl4-(aq) precursors towards the galvanic replacement reaction to synthetize bimetallic AgPd nanoshells, AgAu nanocages, $\mathrm{AgAu}$ triangular nanoframes and $\mathrm{AgAu}$ nanotubes, respectively.

\section{CATALYTIC APPLICATIONS: EFFECT OF SIZE, SHAPE, AND COMPOSITION OVER THEIR ACTIVITIES}

Metallic nanomaterials have been extensively employed catalysis (An and Somorjai 2015,
Fortunelli and Vajda 2016). The importance of these nanostructures in this field mainly comes from the fact that their properties strong correlates to their physical and chemical parameters, which include size (the effect of quantum confinement, as well as the proportions of atoms at corners, edges, and faces), shape (faceting or arrangement of atoms on the surface), composition (monoversus bi- or multimetallic, and the spatial distributions of different elements), and structure (solid, hollow, porous, concave, etc) ( Xia et al. 2009, 2017). During solution phase synthesis, all these parameters can be controlled separately or in combination. Therefore, a deep understanding on how catalytic properties may vary as a function of these above-mentioned parameters is crucial to optimize performance and enable new applications and catalytic behavior. For example, we have investigated the size-dependent optical and catalytic properties of $\mathrm{Au}$ nanospheres towards 4-nitrophenol reduction to form 4-aminophenol as shown in Figure 12 (da Silva et al. 2014). More specifically, the synthesis of aqueous suspensions containing Au NPs $15 \pm 2.2,26 \pm 2.4$, and $34 \pm$ $3.0 \mathrm{~nm}$ in diameter was performed by a seeded growth approach as shown in Figure 12a, c. Then, the catalytic activities as a function of Au NPs size was investigated employing the reduction of 4-nitrophenol. In this case, the reaction kinetics was monitored by UV-VIS spectroscopy in the 350 to $500 \mathrm{~nm}$ range, in which the variations in the absorbance at $400 \mathrm{~nm}$ were monitored as a function of time (Figure 12d, e) (da Silva et al. 2014). The calculated rate constants ( $\mathrm{k}$ ) decreased as the $\mathrm{Au}$ NPs size increased, and corresponded to $0.0150 \pm 0.0008,0.0146 \pm 0.0012$, and 0.0107 $\pm 0.0010 \mathrm{~s}-1$ for $\mathrm{Au}$ NPs $15 \pm 2.2,26 \pm 2.4$, and $34 \pm 3.0 \mathrm{~nm}$ in diameter, respectively. We also employed TOF (turnover frequency) to describe the catalytic activity of Au NPs. Figure $12 \mathrm{f}$ shows the calculated TOF as a function of time for $\mathrm{Au}$ NPs. (da Silva et al. 2014) TOF values decreased 


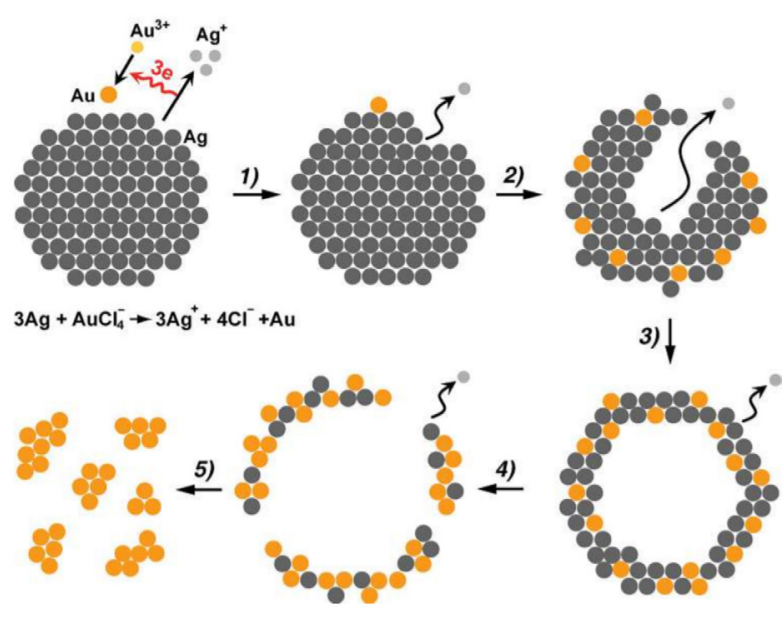

Figure 10 - Schematic illustration of the morphological and structural changes at different stages of the galvanic replacement reaction between a $\mathrm{Ag}$ nanoparticle and $\mathrm{HAuCl}_{4}$ in an aqueous solution. Adapted with permission from ref. (Xia et al. 2013) Copyright 2013 Wiley-VCH.

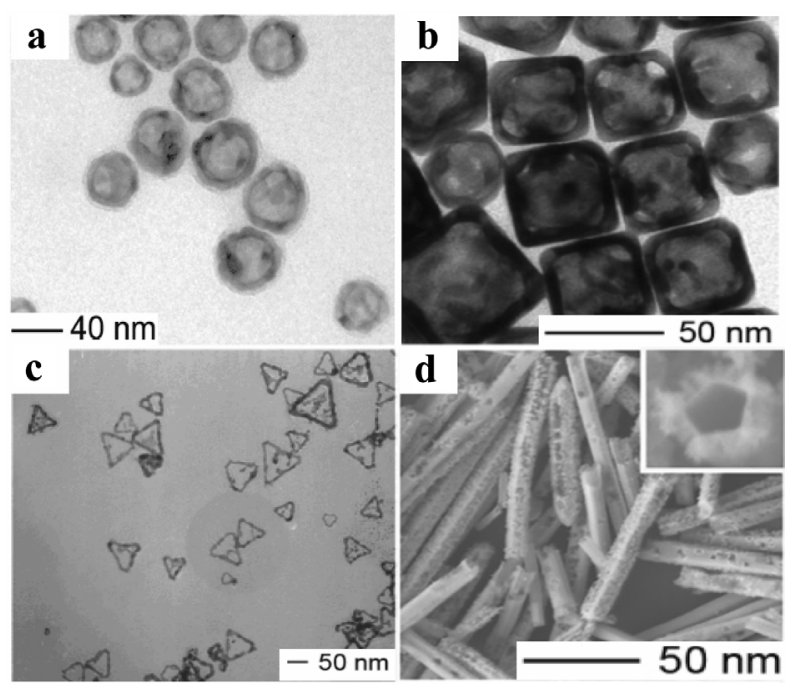

Figure 11 - a-d) TEM images of a) AgPd bimetallic nanoshells, b) $\mathrm{AgAu}$ nanoboxes, c) $\mathrm{AgAu}$ triangular nanoframes, and d) SEM image of AgAu pentagonal nanotubes. Adapted with permission from: a) ref. (Rodrigues et al. 2015) Copyright 2015 Springer Publishing Company, b) ref. (Lu et al. 2007) Copyright 2007 American Chemical Society; c) ref. (Métraux et al. 2003) Copyright 2013 American Chemical Society. d) ref. (Rodrigues et al. 2017) Copyright 2017 Scielo Brazil. with the increase in the Au NPs size, in agreement with the decrease in catalytic activity with size. In this case, while a slight decrease in the TOF values was observed as the Au NPs size increased from 15 to $26 \mathrm{~nm}$, TOFs for Au NPs $34 \mathrm{~nm}$ in diameter were notably smaller. While Au NPs 15 and $26 \mathrm{~nm}$ in diameter reached the maximum TOF at $\sim 26 \mathrm{~s}$, this value corresponded to $91 \mathrm{~s}$ for Au NPs $34 \mathrm{~nm}$ in size, highlighting the importance in controlling nanoparticle sizes for catalytic applications.

Besides the effect of size on the catalytic activity, it has been established that shape of a nanoparticle can also play an important role over catalytic properties, in which reaction rates become dependent on the type of crystallographic facet exposed at the nanoparticle surface (Xia et al. 2017). As a typical case, Chiu et al. performed an evaluation of the catalytic activity of $\mathrm{Au}$ nanoparticles toward the $\mathrm{NaBH} 4$ reduction of p-nitroaniline to p-phenylenediamine at different temperatures (Chiu et al. 2012). The nanocubes, octahedra, and rhombic dodecahedra (Figure 13ac) were employed as catalysts and synthesized by a seed-mediated growth approach, in order to selective expose $\{100\},\{111\}$ and $\{110\}$ facets, respectively. It was found that the catalytic activities for the reduction reaction follows the order of $\{110\}$ $>\{100\}>\{111\}$. Thus, the rhombic dodecahedral gold nanocrystals were found to exhibit the highest reduction rate, followed by those of nanocubes and octahedra in the temperature range analyzed (Figure 13e). (Chiu et al. 2012) Similar results were showed for the catalytic activity in the electrocatalytic and plasmonic activities (Jin et al. 2012, da Silva et al. 2015b)

Another important parameter in catalysis is the effect of composition over their activities. From the perspective of catalysis, a fundamental investigation of how bimetallic nanomaterials can improve catalytic activities is crucial for engineering nanostructures with high performances for a target application (Gilroy et al. 2016). In this 

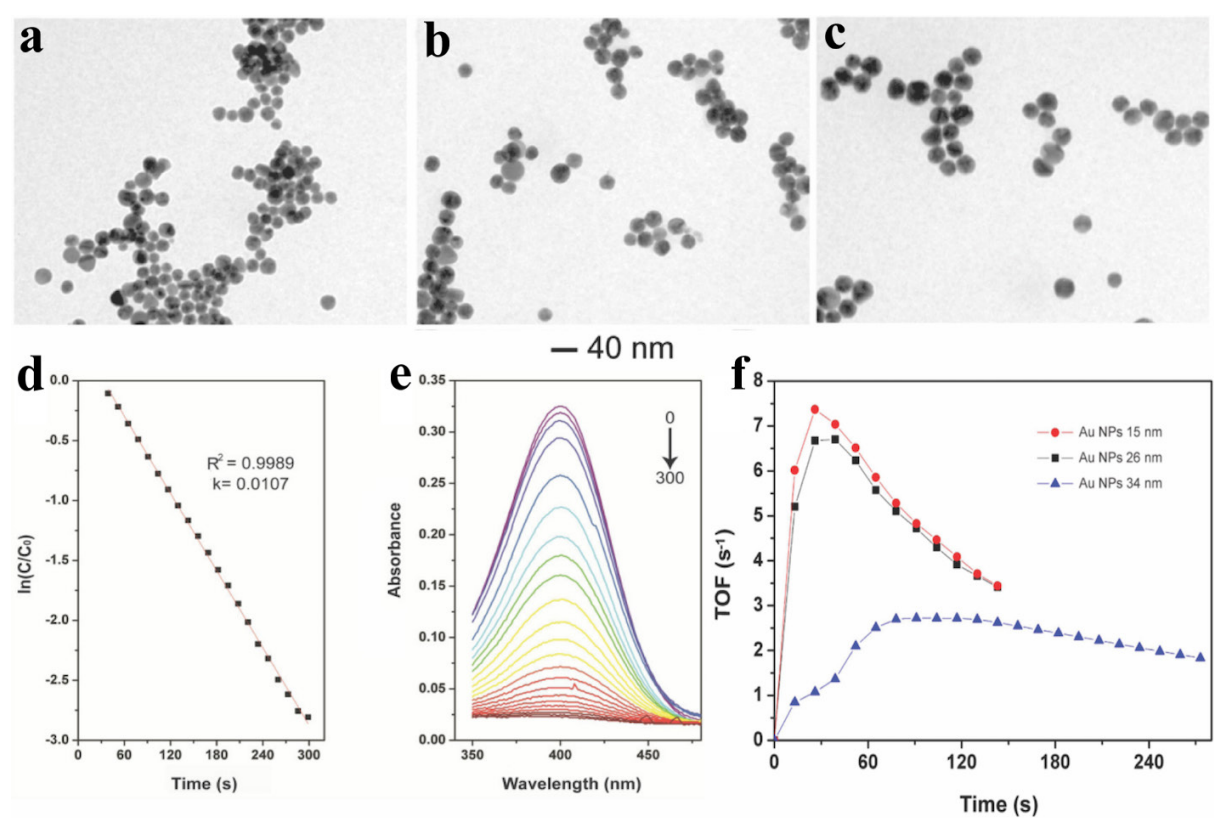

Figure 12 - TEM images of Au nanospheres with a) 15, b) 26 and c) $34 \mathrm{~nm}$, respectively. d) Plot of $\ln \left(\mathrm{C} / \mathrm{C}_{0}\right)$ and $\left.\mathbf{e}\right) \mathrm{UV}$-Vis spectra recorded as a function of time at room temperature for $\mathrm{Au}$ nanospheres with $34 \mathrm{~nm}$ in diameter. f) Calculated TOFs as a function of time for the 15, 26 and $34 \mathrm{~nm}$ Au nanospheres (red, black and blue traces, respectively). Adapted with permission from ref. (da Silva et al. 2014) Copyright 2014 Scielo Brazil.
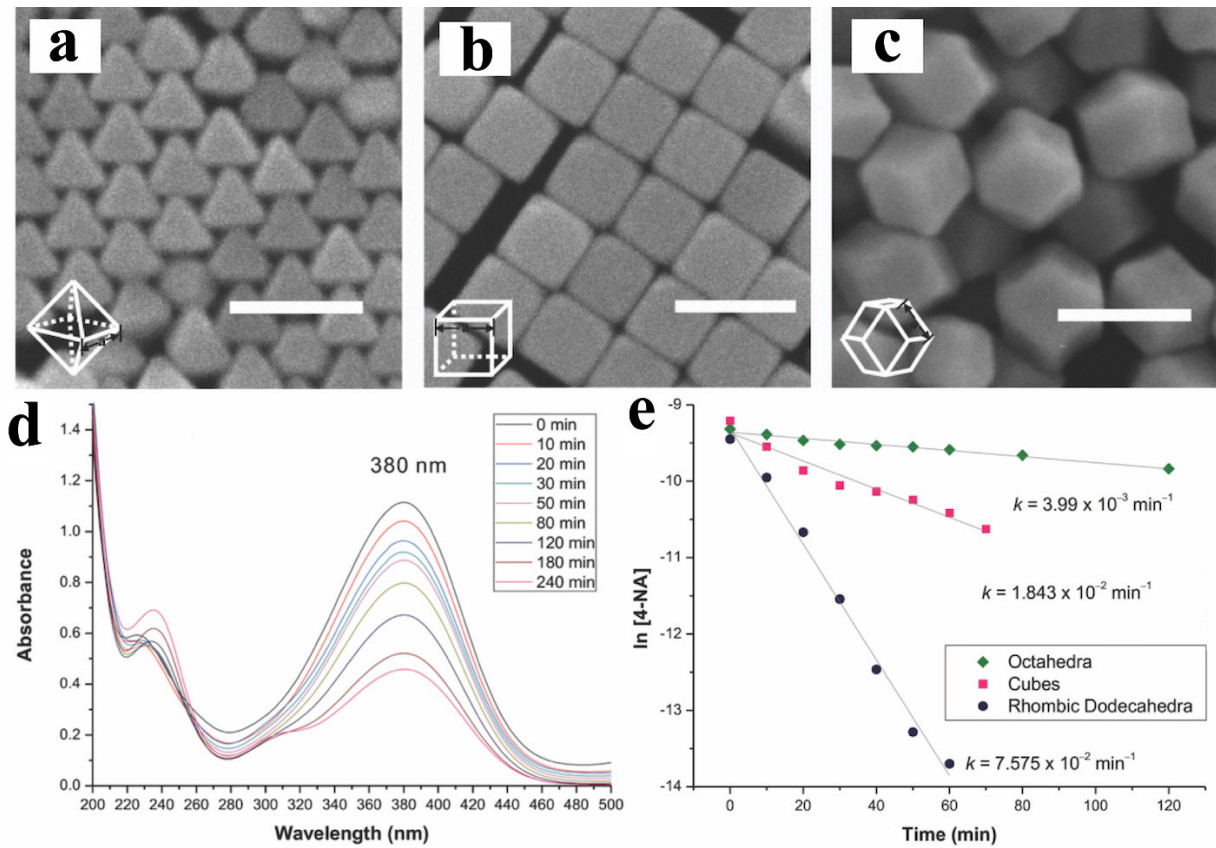

Figure 13 - SEM images of a) Au octahedra, b) Au nanocubes, and c) Au rhombic dodecahedra. Scale bars are equal to $100 \mathrm{~nm}$. d) Time-dependent UV-vis absorption spectra for the borohydride reduction of 4-aminophenol (4-NA) at $25{ }^{\circ} \mathrm{C}$ and e) $\ln (4-\mathrm{NA})$ versus time plots using gold nanocubes, octahedra, and rhombic dodecahedra as catalysts (green, red and black points, respectively). Adapted with permission from ref. (Chiu et al. 2012) Copyright 2012 American Chemical Society. 
case, it has been demonstrated that two main key factors are related to the higher catalytic activities of bimetallic nanomaterials with controllable compositions: (i) the electronic and (ii) geometric effects (Gilroy et al. 2016, da Silva et al. 2017). The electronic effect has been described as changes in the electronic structure of a nanomaterial during the formation of a bimetallic particle relative to the monometallic counterparts (Kim et al. 2014). In this case, the way their d-band interacts with the molecules/adsorbates determines their binding strength. In general, the more low-lying (relative to the Fermi level) the d-band, the weaker the binding due to the occupancy of antibonding states (Kim et al. 2014). The geometric effect is associated with the shape of the bimetallic nanostructures, which determines the atomic arrangement at the surface of a nanocatalyst (Kim et al. 2014). This arrangement at the surface can significantly vary as function of the composition, which also lead to a significant effect on the binding strength of substrates and products. For example, Stamenkovic et al synthesized pure platinum-nickel single-crystals of alloys with different atomic arrangements of their crystalline lattices (Stamenkovic et al. 2007). They compared the samples with single crystals of pure platinum as well as with conventional platinum-carbon fuel cell catalysts. They concluded that the most tightly packed arrangement of atoms, the $\{111\}$ surface, were much more active relative those conventional and monometallic materials. Moreover, platinumnickel bimetallic single-crystals lead to an activity increase of 10 and 90 folds relative to single-crystal $\mathrm{Pt}$ and standard $\mathrm{Pt} / \mathrm{C}$ materials (Stamenkovic et al. 2007).

\section{CATALYTIC APPLICATIONS: CONTROLLED NANOPARTICLES SUPPORTED IN METAL OXIDES}

Heterogeneous catalysis represents a crucial discipline when it comes to providing the scientific and technological foundation for making cleaner, more efficient, and economically viable chemical production processes. These comprise essential requisites towards enabling a sustainable future (Mizuno and Misono 1998, Norskov et al. 2008, Schlögl 2015). Heterogeneous catalysis is also one of the most relevant topics in the industrial scenario, leading advances sustainability by improving the exploitation of raw materials, enabling the transition from fossil to renewable feedstocks, reducing energy consumption, and minimizing the environmental footprint. Moreover, this field also plays a central role in the fields of transportation and health, encompassing the development of new fuels and devices (such as fuel cells), synthetic biology, and the synthesis of bioactive molecules such as antibiotics (Dincer 2007, Polshettiwar and Varma 2010, Wells and Meyer 2014). As a matter of fact, because catalysis is involved in the synthesis of over $85 \%$ of all chemical products produced today, new developments in catalysis is expected to have a huge impact addressing the several challenges currently faced by humankind (Mizuno and Misono 1998, Norskov et al. 2008, Wells and Meyer 2014, Schlögl 2015).

Heterogeneous catalysts can be defined as structures that alter the rate of a chemical reaction by modifying several mechanistic steps relative to the noncatalyzed reaction (usually leading to a reduction of its activation energy). The catalyst is also recovered or unchanged after the reaction finishes (Nørskov et al. 2014, Albonetti et al. 2015, Corma 2016). They are normally comprised of active sites (usually transition metals) deposited at the surface of a solid support that will change/ increase the rate of a target chemical reaction (Norskov et al. 2008, Schlögl 2015, Corma 2016). They usually display high surface areas (200-1600 $\left.\mathrm{m}^{2} \mathrm{~g}^{-1}\right)$, and it is desirable that the number of active sites per unit of volume is maximized (LePage et al. 1997, Mizuno and Misono 1998). However, the availability of well-established methods/protocols that are effective for producing highly active, stable, 
and recyclable catalysts that are economically viable remains limited (Astruc et al. 2005, Norskov et al. 2008, Corma 2016).

The enormous interest in the utilization of metal nanoparticles as catalysts (a field also named as nanocatalysis) is mainly motivated by their high surface areas, which increase the number of available active sites at the catalyst surface, favoring effective interactions between the substrates and the catalyst surface. This has led to high activities towards relevant chemical transformations such as oxidations, reductions, and $\mathrm{C}-\mathrm{C}$ coupling reactions, even under mild conditions (Fihri et al. 2011, Albonetti et al. 2015, An and Somorjai 2015). These qualities are traditionally assigned to homogeneous catalysts. However, metal nanoparticles are not soluble in the reaction mixture. Therefore, nanocatalysts have bridged the gap between homogeneous and heterogeneous catalysts, uniting the advantages of both approaches (Fihri et al. 2011, Albonetti et al. 2015, An and Somorjai 2015). Another crucial feature is that metal nanoparticles provide distinctive opportunities for the optimization and maximization of catalytic performances via controlled synthesis.

Despite these attractive features, several challenges remain regarding the use of metal nanostructures as catalysts. One key issue is the availability of synthetic strategies that are simple, robust, and scalable for generating controlled metal nanoparticles (Fihri et al. 2011, Albonetti et al. 2015, An and Somorjai 2015). Even if this is achieved, the particles must be supported to produce materials displaying a uniform and precise distribution of nanoparticles/active sites over the support. Thus, another current challenge in catalysis by noble metals comprises the generation of supported materials in which the metal component presents a uniform dispersion over the entire surface of the support (without agglomeration) (Rodrigues et al. 2016b). In this regard, the synthesis of supported state-of-the art catalysts is often characterized by poor control over size, shape, composition, and distribution of the noble metal component over the support (Freakley et al. 2015).

Due to the aforementioned drawbacks, progress in the field of catalysis, in general, has mainly occurred via collected knowledge, trial and error, and interpretation of results (Corma 2016). In this context, the development of controlled nanocatalysts is a must towards the design of catalysis with desired performances. Many methods are usually employed for their syntheses. The conventional synthesis of supported state-ofthe-art catalysts is performed by impregnation, precipitation, deposition/ precipitation, sol-gel, colloidal, and hydrothermal approaches, which often do not enable a precise control over size, shape, composition, and distribution of the noble metal component over the support (Hutchings and Kiely 2013, An and Somorjai 2015, Gilbertson et al. 2015, Sharma et al. 2015).

In order to address the above-mentioned challenges, enormous advances have been directed towards the synthesis of supported nanoparticles consisting of truly controlled nanomaterials based on $\mathrm{Ag}, \mathrm{Au}, \mathrm{Pd}, \mathrm{Pt}, \mathrm{Ru}, \mathrm{Rh}$, and Ir. This is imperative for generating fundamental knowledge on the understanding of how catalytic properties depend on the various parameters that define a nanocatalyst (Freakley et al. 2015, Gilbertson et al. 2015, Corma 2016, Gilroy et al. 2016, Xia et al. 2017). For example, our group demonstrated a facile approach to decorate $\mathrm{TiO} 2$ with $\mathrm{Au}$ nanoparticles displaying well-defined sizes and uniform deposition (Figure 14) by successive reduction steps (Damato et al. 2013). In this study, Au nanospheres were generated over the $\mathrm{TiO} 2$ supporting material $(\sim 220$ $\mathrm{nm}$ in diameter) by successive reduction steps, employing AuCl4- (aq) and ascorbic acid. This approach enabled the growth of Au nanospheres from $12.2 \mathrm{~nm}$ up to 19.3 in the second step and 24.5 in the final step, conserving their optical properties, 

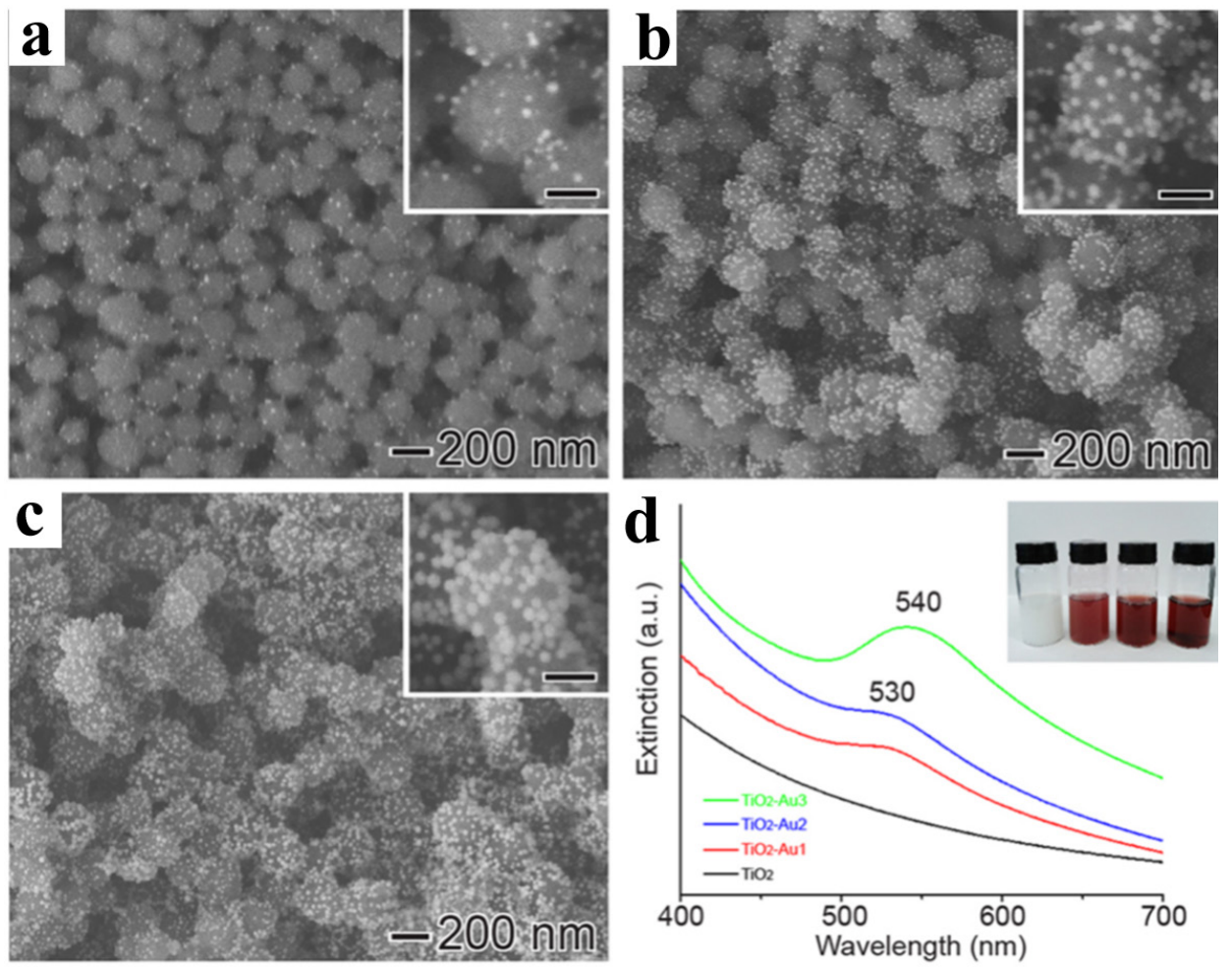

Figure 14 - a-c) SEM images of $\mathrm{TiO}_{2}$-Au colloidal spheres that were obtained after the first a), second b), and third c) reduction steps employing $3 \mathrm{~mL}$ of $1 \mathrm{mM} \mathrm{AuCl}_{4(\mathrm{aq})}^{-}$as the precursor solution. The scale bars in the insets correspond to $100 \mathrm{~nm}$. d) UV-vis extinction spectra recorded for the $\mathrm{TiO}_{2}$ (black trace) and $\mathrm{TiO}_{2}-\mathrm{Au}$ (red, blue ang green traces correspond to samples showed in $\mathbf{a}, \mathbf{b}$, and $\mathbf{c}$, respectively). The inset depicts a photograph of $\mathrm{TiO}_{2}$, and samples $\mathbf{a}, \mathbf{b}$ and $\mathbf{c}$, aqueous suspensions (from left to right, respectively). Adapted with permission from ref. (Damato et al. 2013) Copyright 2013 American Chemical Society.

morphology, and dispersions. Here, it has been shown that smaller Au nanospheres were better for this type of reaction due to a significant difference in surface-to-volume ratio between samples.

Recently, we have reported the optimization of both the support and the active phase to obtain highly active catalysts towards green oxidations. We developed $\mathrm{MnO} 2$ nanowires decorated with ultrasmall Au NPs catalysts that displayed large specific surface areas, ultrasmall and monodisperse $\mathrm{Au}$ NPs sizes, no agglomeration, strong metalsupport interactions, and high concentration of oxygen vacancies and $A u \delta+$ species at their surface as shown in Figure 15 (da Silva et al. 2016). We showed that high catalytic performances $(\mathrm{TOF}=$ $590000 \mathrm{~h}-1$ ) could be achieved towards the green oxidation of silanes and $\mathrm{H} 2$ production under ultralow Au loadings (0.001-0.0002 $\mathrm{mol} \%$ in terms of $\mathrm{Au}$ ) employing $\mathrm{H} 2 \mathrm{O}$ as the oxidant, $25{ }^{\circ} \mathrm{C}$ as the reaction temperature, and $\mathrm{MnO} 2$ nanowires decorated with ultrasmall Au NPs $(3 \mathrm{~nm})$ as catalysts (da Silva et al. 2016). In addition to these high activities towards a variety of substrates, the MnO2-Au NPs displayed good stability/ recyclability, in which no morphological changes or loss of activity were observed even after 10 reaction cycles (da Silva et al. 2016).

Among the different strategies that have been described for the synthesis of supported and controlled catalysts, the galvanic replacement reaction is especially attractive as it allows the formation of uniform, bimetallic and hollow 


\section{a}
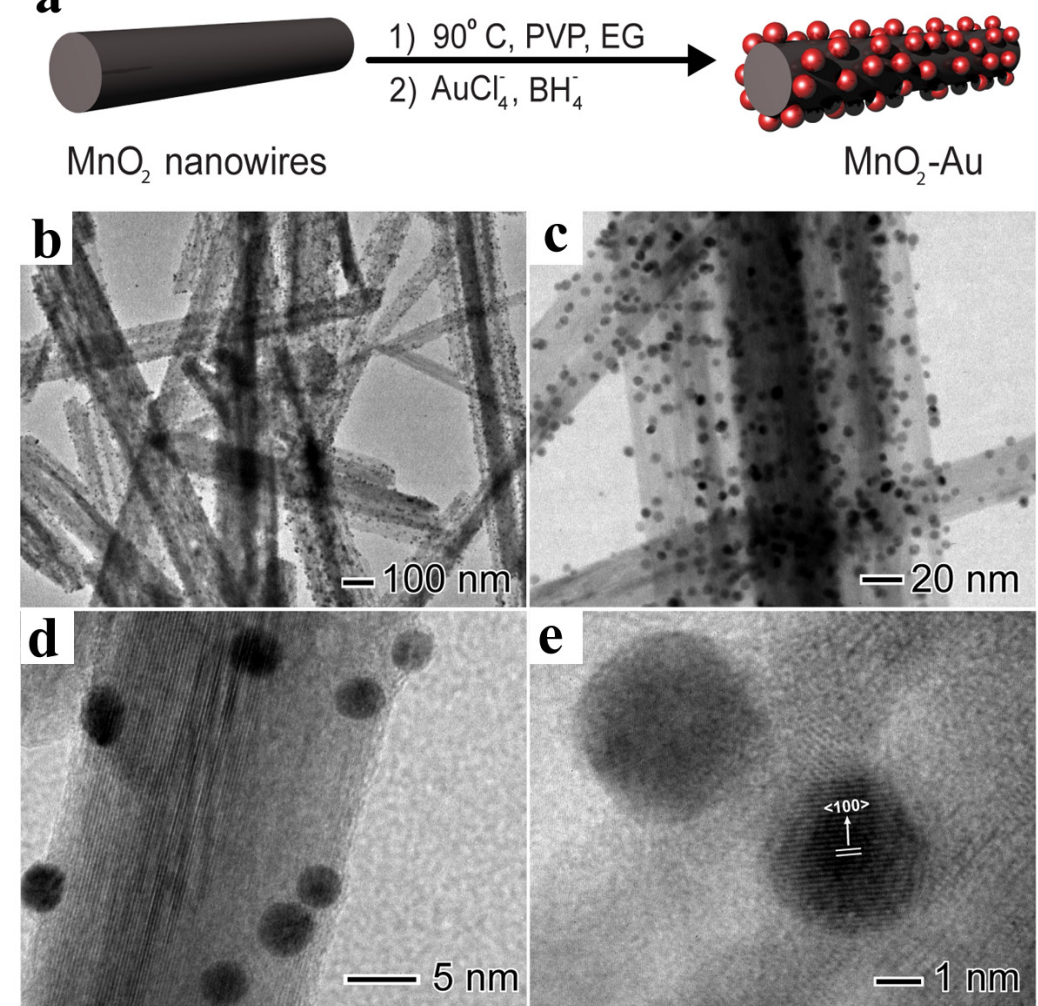

Figure 15 - a) Strategy for the synthesis of $\mathrm{MnO}_{2}$ nanowires decorated with ultrasmall $\mathrm{Au}$ NPs. b-e) HRTEM images of $\mathrm{MnO}_{2}-\mathrm{Au}$ NPs depicting the ultrasmall and monodisperse $\mathrm{Au}$ NPs size as well as their uniform distribution over the $\mathrm{MnO}_{2}$ surface. The lattice fringe orientations in the phase-contrast HRTEM images (d and e) show that both the $\mathrm{MnO}_{2}$ nanowires and $\mathrm{Au}$ NPs were single crystalline. Adapted with permission from ref. (da Silva et al. 2016) Copyright 2016 Elsevier.

nanomaterials displaying ultrathin walls in a onestep reaction, using water as the solvent, and in short reaction times (Cobley and Xia 2010, Xia et al. 2013, El Mel et al. 2016, da Silva et al. 2017). These nanostructures can be easily deposited on the surface of inorganic supports $(\mathrm{SiO} 2, \mathrm{TiO} 2, \mathrm{Al} 2 \mathrm{O} 3$, etc) by a wet-impregnation approach, leading to the formation of controlled supported nanocatalysts (Jiang 2006, da Silva et al. 2017). We recently have demonstrated that our described approach combining galvanic replacement reactions and coreduction in the presence of hydroquinone allowed us to synthesize $\mathrm{AgAu}, \mathrm{AgPd}$, and $\mathrm{AgPt}$ hollow nanomaterials with controlled compositions, sizes, and surface morphologies (da Silva et al. 2015a, b, Rodrigues et al. 2016a, b). These nanomaterials could then be employed to obtain supported catalysts at the gram-scale after their attachment to commercial silica. Figure 16 shows SEM images of $\mathrm{AgAu}$ nanodendrites with controlled surface morphologies supported on commercial $\mathrm{SiO} 2$ by this above-mentioned method (da Silva et al. 2015a). The size and surface morphology of the nanodendrites was found to directly affect their catalytic activity towards the gas-phase oxidation of benzene, toluene, and o-xylene, which is an important reaction for the removal of these toxic compounds from fuels and for environmental remediation. All produced nanodendrite particles were found to be catalytically active, even at low temperatures and low metal loadings (da Silva et al. 2015a). 
In addition to optimizing and controlling catalytic properties by manipulating the various physical and chemical parameters that define a nanocatalyst, plasmonically-enhanced or plasmonically-mediated transformations (SPRenhanced or SPR-mediated catalysis) represents a new frontier in the field of heterogeneous catalysis (Catchpole and Polman 2008, Christopher et al. 2011, Sun and Xu 2012, Baffou and Quidant 2014, Wang et al. 2014, Kang et al. 2015). Here, visible light can be employed as a sustainable energy input to drive and control chemical reactions. Plasmonic nanostructures, such as silver (Ag) and gold $(\mathrm{Au}) \mathrm{NPs}$, can strongly interact with visible or near-infrared light, as a result, the SPR excitation (Linic et al. 2011, Sun and Xu 2012, Baffou and Quidant 2014). In the context of catalysis, the SPR excitation can be put to work for enhancing or mediating chemical transformations by several mechanisms as expressed in Figure 17 (Linic et al. 2011, Sun and Xu 2012, Baffou and Quidant 2014). These include: i) by increasing the local electric field close to the nanostructure surface that enhances absorption at the metal-molecule interface; ii) by generating electron-hole pairs that enable the occurrence of a charge-transfer process at the metal-molecule interface; and iii) by generating local heating due to plasmon decay at the metal surface that provides energy input for some conversions. It is noteworthy that, while heat is a major parameter in any chemical reaction, light can be used to achieve high selectivity in chemical mechanisms thanks to quantum selection rules and adjustable photon energies. Moreover, electron transfer is the basis of redox reactions. Hence, the idea to use metal NPs as efficient sources of heat, light, and electrons appears to be an appealing and intuitive concept to both improve yields and control of chemical reactions.

Generally, it is believed that the improved matching between the SPR and excitation wavelength may lead to higher SPR-mediated
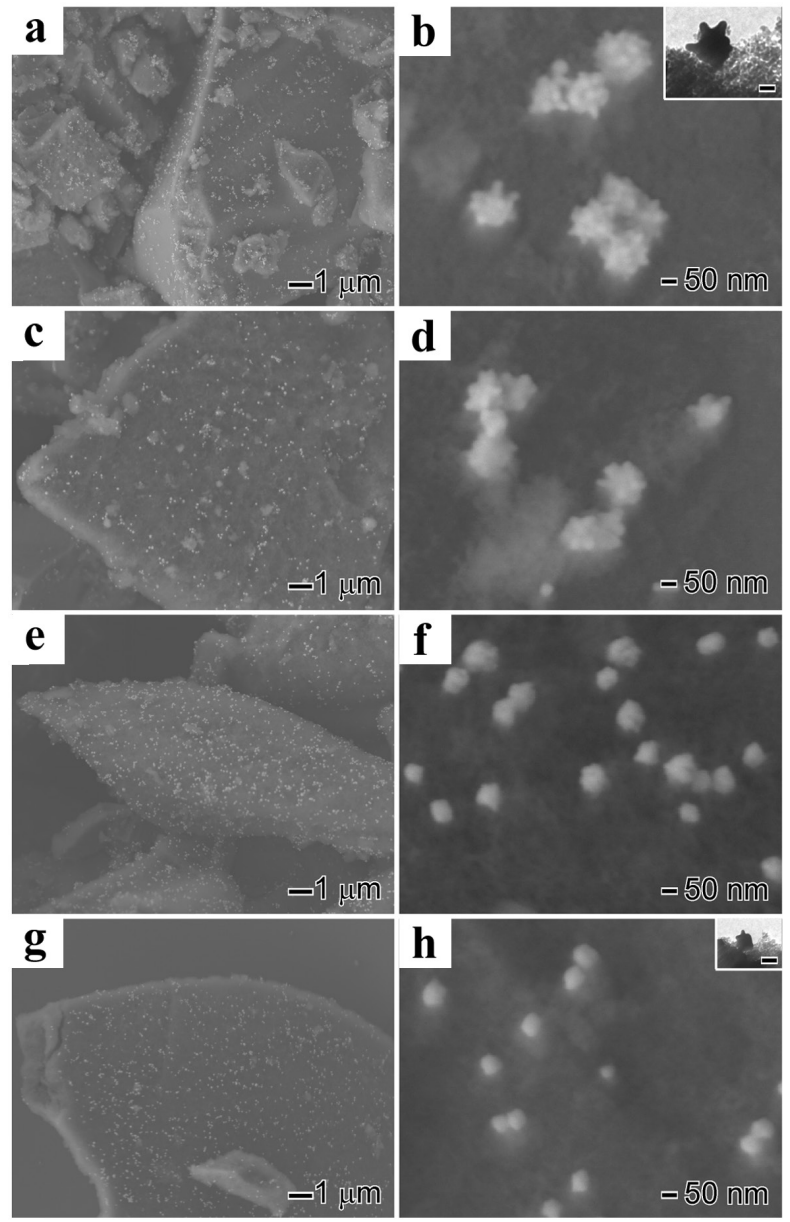

Figure 16 - SEM images of AgAu nanodendrites with controlled surface morphologies supported on commercial $\mathrm{SiO}_{2}\left(\mathrm{AgAu} / \mathrm{SiO}_{2}\right)$ by wet impregnation. The average sizes of the AgAu nanodendrites corresponded to: (a and b) $148 \pm 6$ $\mathrm{nm}$, (c and d) $96 \pm 5 \mathrm{~nm}$, (e and f) $66 \pm 4 \mathrm{~nm}$, and (g and h) $45 \pm$ $3 \mathrm{~nm}$. Adapted with permission from ref. (da Silva et al. 2015a) Copyright 2015 American Chemical Society.

catalytic activities (Sun and Xu 2012, Baffou and Quidant 2014, Rodrigues et al. 2016b). However, a better understanding of the factors that govern SPR-mediated processes is imperative to the rational design of optimized catalysts for target transformations. For example, systematic investigations on the effect of the particle size, shape, composition, and crystalline structure of the activities are still scarce. In this context, we found that the composition of bimetallic $\mathrm{AgAu}$ nanoparticles plays an important role in the SPRmediated catalytic activity (Wang et al. 2014). We 
$\mathbf{a}$

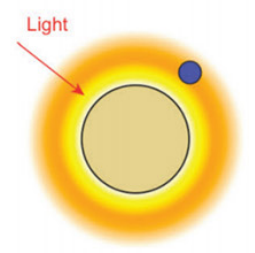

b

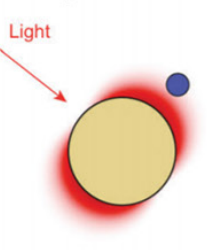

c

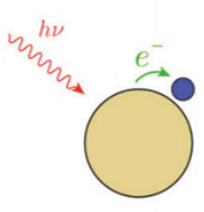

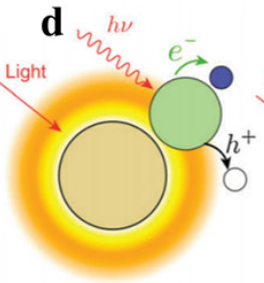

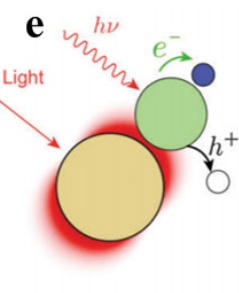

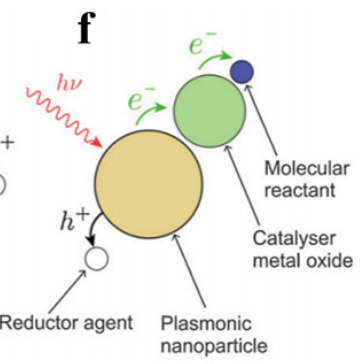

Figure 17 - Main mechanisms involved in plasmonic catalysis: a) photo-induced temperature increase provides heat to an adjacent reactant; b) the enhancement of the optical near field at the vicinity of the NP increases the photon rate seen by an adjacent reactant; c) a photoinduced hot electron is transferred to a nearby reactant; $\mathbf{d})$ the electron-hole $\left(\mathrm{e}^{-}-\mathrm{h}^{+}\right)$generation rate in a photocatalyst is enhanced by heat generated by the NP; e) the electron-hole generation rate in a photocatalyst is enhanced by the strong optical near-field of the plasmonic NP; and f) the photocatalyst adjacent to the NP is activated by hot electron transfer from the plasmonic NP. Adapted with permission from ref. (Baffou and Quidant 2014) Copyright 2014 Royal Society of Chemistry.
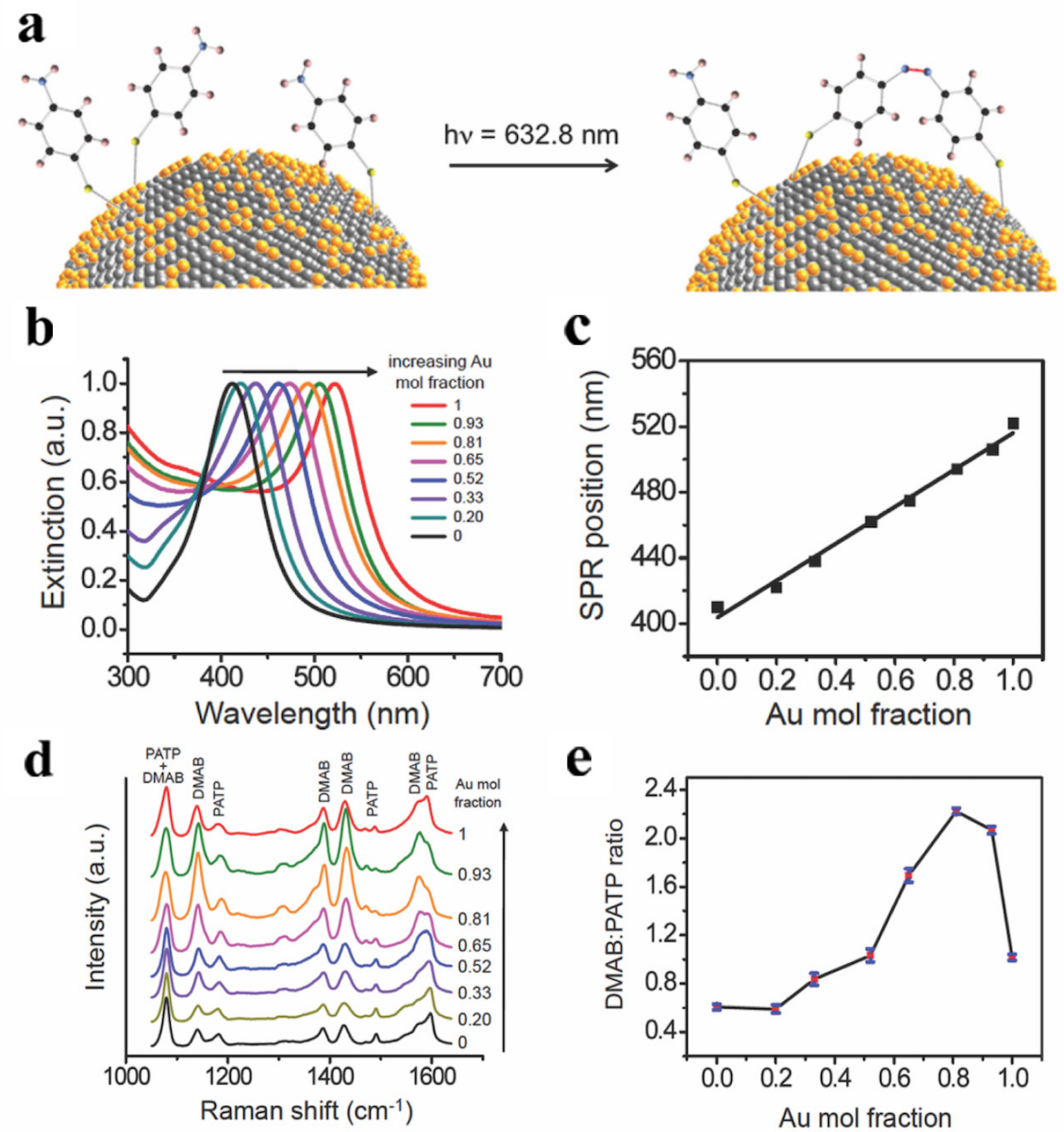

c

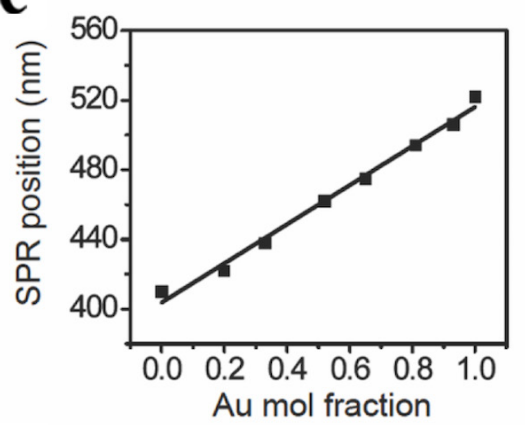

$\mathbf{e}$

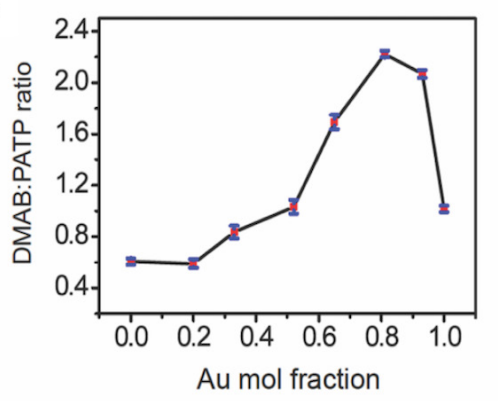

Figure 18 - a) Scheme showing the SPR-mediated oxidation of PATP to DMAB by ${ }^{3} \mathrm{O}_{2}$ employing AgAu nanoparticles as catalysts; b) UV-Vis extinction spectra and c) SPR peak positions as a function of the Au content (Au mole fraction) in the AgAu nanoparticles; d) SERS spectra as a function of composition (Au mole fraction) employing Ag, AgAu and $\mathrm{Au}$ nanoparticles functionalized with PATP; e) Obtained DMAB/PATP 1433/1593 $\mathrm{cm}^{-1}$ intensity ratios as a function of the Au content in the nanoparticles. Adapted with permission from ref. (Wang et al. 2014) Copyright 2014 American Chemical Society. 
investigated the SPR-mediated activities of AgAu alloyed nanospheres towards the oxidation of p-aminothiopheol to p,p'-dimercaptoazobenzene (Figure 18a) (Wang et al. 2014). By changing the nanoparticles composition, they were able to redshift the main plasmonic peak form $\sim 400$ to $\sim 525 \mathrm{~nm}$ (Figure 18b-c) (Wang et al. 2014). The SPR-mediated reaction was conducted using a Raman spectrometer employing a laser irradiation of $632.8 \mathrm{~nm}$ as source as well as to monitor the product conversion by the SERS effect. As can be seen in Figure 18e a volcano-type relationship between the AgAu chemical composition and catalytic performance of the obtained nanoparticles was observed, with a maximum activity for the Ag0.19Au0.81 composition (Wang et al. 2014). This behavior can be by the balance between the matching of excitation laser wavelength and the SPR position, that lead to stronger plasmonic excitations and the damping of plasmon oscillation due to $\mathrm{Au}$ interband transitions above $\sim 500 \mathrm{~nm}$ (Wang et al. 2014).

\section{CONCLUSIONS}

This review summarized the definitions, recent studies, and challenges involved in the controlled synthesis of noble metal nanoparticles. It is clear that the literature concerning the controlled synthesis of metal nanomaterials continues to expand. It is noteworthy that the basis for the experimental design of controlled nanomaterials is transitioning from the conventional trial and error method (empirical strategy) toward well-founded, designed-driven theory. Several advances have been achieved over the past decade towards the fundamental understanding of thermodynamics and kinetics that guide atoms, clusters or molecules to assemble in controlled nanomaterials. Enormous progress in this field was only possible due to the mechanistic understanding of the controlled synthesis of metal nanomaterials in solution, in which nucleation and growth pathways are crucial for tuning obtained sizes, shapes, compositions, and architectures. Finally, we discussed the unique optical and catalytic applications associated with noble metal nanoparticles, highlighting some recent examples to highlight how the use of controlled nanomaterials for heterogeneous catalysis and plasmon-enhanced catalysis is important and can lead to improved performances/activities. We believe this review will not only assist scientists in understanding the controlled synthesis of metal nanomaterials but also may inspire the development of new and more sophisticated metal nanomaterials with improved or target performances for the desired application.

\section{ACKNOWLEDGMENTS}

This work was supported by the Fundação de Amparo à Pesquisa do Estado de São Paulo (FAPESP) (grant numbers 2015/21366-9 and 2015/26308-7). Pedro H.C. Camargo thanks Conselho Nacional de Desenvolvimento Científico e Tecnológico for a research fellowship. Rafael S. Geonmonond and Anderson G.M. da Silva thank Coordenação de Aperfeiçoamento de Pessoal de Nível Superior (CAPES) and Conselho Nacional de Desenvolvimento Científico e Tecnológico (CNPq) for the fellowships. The authors gratefully acknowledge corresponding publishers for the kind permissions to reproduce their materials, especially figures, used in this review article.

\section{REFERENCES}

ALBONETTI S, MAZZONI RAND CAVANI F. 2015. Chapter 1 Homogeneous, Heterogeneous and Nanocatalysis. In: Transition Metal Catalysis in Aerobic Alcohol Oxidation. Cambridge: Royal Society of Chemistry, p. 1-39.

AN K AND HYEON T. 2009. Synthesis and biomedical applications of hollow nanostructures. Nano Today 4: 359373.

AN K AND SOMORJAI GA. 2015. Nanocatalysis I: Synthesis of Metal and Bimetallic Nanoparticles and Porous Oxides 
and Their Catalytic Reaction Studies. Catal Lett 145: 233248.

ARICO AS, BRUCE P, SCROSATI B, TARASCON JM AND VAN SCHALKWIJK W. 2005. Nanostructured materials for advanced energy conversion and storage devices. Nat Mater 4: 366-377.

ASTRUC D, LU F AND ARANZAES JR. 2005. Nanoparticles as Recyclable Catalysts: The Frontier between Homogeneous and Heterogeneous Catalysis Angewandte 44: 7852-7872.

BAFFOU G AND QUIDANT R. 2014. Nanoplasmonics for chemistry. Chem Soc Rev 43: 3898-3907.

BARNARD A S, YOUNG NP, KIRKLAND AI, VAN HUIS MA AND XU H. 2009. Nanogold: A Quantitative Phase Map. ACS Nano 3: 1431-1436.

BISWAS A, BAYER IS, BIRIS AS, WANG T, DERVISHI E AND FAUPEL F. 2012. Advances in top-down and bottom-up surface nanofabrication: Techniques, applications $\{\&\}$ future prospects. Adv Colloid Interface Sci 170: 2-27.

CAMARGO PHC, RODRIGUES TS, DA SILVA AGM AND WANG J. 2015. Controlled Synthesis: Nucleation and Growth in Solution. Metallic Nanostructures: From Controlled Synthesis to Applications. In: Xiong Y and Lu X (Eds), Springer International Publishing: Cham, p. 49-74.

CATCHPOLE KR AND POLMAN A. 2008. Plasmonic solar cells. Opt Express 16: 21793-21800.

CHEN J, LIM B, LEE EP AND XIA Y. 2009. Shape-controlled synthesis of platinum nanocrystals for catalytic and electrocatalytic applications. Nanotoday 4(1): 81-95.

CHEN L, JI F, XU Y, HE L, MI Y, BAO F, SUN B, ZHANG $X$ AND ZHANG Q. 2014. High-Yield Seedless Synthesis of Triangular Gold Nanoplates through Oxidative Etching. Nano Lett 14: 7201-7206.

CHIU CY, CHUNG PJ, LAO KU, LIAO CW AND HUANG MH. 2012. Facet-Dependent Catalytic Activity of Gold Nanocubes, Octahedra, and Rhombic Dodecahedra toward 4-Nitroaniline Reduction. J Phys Chem C 116: $23757-$ 23763.

CHRISTOPHER P, XIN H AND LINIC S. 2011. Visible-lightenhanced catalytic oxidation reactions on plasmonic silver nanostructures. Nat Chem 3: 467-472.

COBLEY CM, SKRABALAK SE, CAMPBELL DJ AND XIA Y. 2009. Shape-Controlled Synthesis of Silver Nanoparticles for Plasmonic and Sensing Applications. Plasmonics 4: 171-179.

COBLEY CM AND XIA Y. 2010. Engineering the properties of metal nanostructures via galvanic replacement reactions. Mater Sci Eng R Rep 70: 44-62.

CORMA A. 2016. Heterogeneous Catalysis: Understanding for Designing, and Designing for Applications. Angew Chem Int Edit 55: 6112-6113.
DA SILVA AGM, RODRIGUES TS, MACEDO A, SILVA RTP DA AND CAMARGO PHC. 2014. An undergraduate level experiment on the synthesis of $\mathrm{Au}$ nanoparticles and their size-dependent optical and catalytic properties. Quím Nova 37: 1716-1720.

DA SILVAAGM ET AL. 2015a. Controlling Size, Morphology, and Surface Composition of AgAu Nanodendrites in $15 \mathrm{~s}$ for Improved Environmental Catalysis under Low Metal Loadings. ACS Applied Materials \& Interfaces 7: 2562425632.

DA SILVA AGM, RODRIGUES TS, WANG J, YAMADA LK, ALVES T V, ORNELLAS FR, ANDO RA AND CAMARGO PHC. 2015b. The Fault in Their Shapes: Investigating the Surface-Plasmon-Resonance-Mediated Catalytic Activities of Silver Quasi-Spheres, Cubes, Triangular Prisms, and Wires. Langmuir 31: 10272-10278.

DA SILVA AGM ET AL. 2016. MnO2 nanowires decorated with Au ultrasmall nanoparticles for the green oxidation of silanes and hydrogen production under ultralow loadings. Appl Catal B-Environ 184: 35-43.

DA SILVA AGM, RODRIGUES TS, HAIGH SJ AND CAMARGO PHC. 2017. Galvanic replacement reaction: recent developments for engineering metal nanostructures towards catalytic applications. Chem Commun 53: 71357148.

DAMATO TC, DE OLIVEIRA CCS, ANDO RA AND CAMARGO PHC. 2013. A Facile Approach to TiO2 Colloidal Spheres Decorated with Au Nanoparticles Displaying Well-Defined Sizes and Uniform Dispersion. Langmuir 29: 1642-1649.

DINCER I. 2007. Environmental and sustainability aspects of hydrogen and fuel cell systems. Int J Energy Res 31: 29-55.

EL MEL AA ET AL. 2016. Galvanic Replacement Reaction: A Route to Highly Ordered Bimetallic Nanotubes. J Phys Chem C 120: 17652-17659.

EUSTIS S AND EL-SAYED MA. 2006. Why gold nanoparticles are more precious than pretty gold: Noble metal surface plasmon resonance and its enhancement of the radiative and nonradiative properties of nanocrystals of different shapes. Chem Soc Rev 35: 209-217.

FARADAY M. 1857. The Bakerian Lecture: Experimental Relations of Gold (and Other Metals) to Light. Phylosophical Transactions 147: 145-181.

FIHRI A, BOUHRARA M, NEKOUEISHAHRAKI B, BASSET JM AND POLSHETTIWAR V. 2011. Nanocatalysts for Suzuki cross-coupling reactions. Chem Soc Rev 40: 5181-5203.

FORTUNELLI A AND VAJDA S. 2016. Editorial: Nanocatalysis. Catal Sci Technol 6: 6763-6765.

FREAKLEY SJ, QIAN H, KIELY CJ AND HUTCHINGS GJ. 2015. Gold Catalysis: A Reflection on Where We are Now. Catal Lett 145: 71-79. 
GILBERTSON LM, ZIMMERMAN JB, PLATA DL, HUTCHISON JE AND ANASTAS PT. 2015. Designing nanomaterials to maximize performance and minimize undesirable implications guided by the Principles of Green Chemistry. Chem Soc Rev 44: 5758-5777.

GILROY KD, PENG HC, YANG X, RUDITSKIY A AND XIA Y. 2017. Symmetry breaking during nanocrystal growth. Chem Commun 53: 4530-4541.

GILROY KD, RUDITSKIY A, PENG HC, QIN D AND XIA Y. 2016. Bimetallic Nanocrystals: Syntheses, Properties, and Applications. Chem Rev 116: 10414-10472.

GRANASY L, PODMANICZKY F, TOTH GI, TEGZE G AND PUSZTAI T. 2014. Heterogeneous nucleation of/on nanoparticles: a density functional study using the phasefield crystal model. Chem Soc Rev 43: 2159-2173.

HUTCHINGS GJ AND KIELY CJ. 2013. Strategies for the Synthesis of Supported Gold Palladium Nanoparticles with Controlled Morphology and Composition. Acc Chem Res 46: 1759-1772.

JAIN PK, HUANG X, EL-SAYED IH AND EL-SAYED MA. 2007. Review of Some Interesting Surface Plasmon Resonance-enhanced Properties of Noble Metal Nanoparticles and Their Applications to Biosystems. Plasmonics 2: 107-118.

JARIWALA D, SANGWAN VK, LAUHON LJ, MARKS TJ AND HERSAM MC. 2013. Carbon nanomaterials for electronics, optoelectronics, photovoltaics, and sensing. Chem Soc Rev 42: 2824-2860.

JIANG SP. 2006. A review of wet impregnation - An alternative method for the fabrication of high performance and nanostructured electrodes of solid oxide fuel cells. Mater Sci Eng A 418: 199-210.

JIN M, ZHANG H, XIE Z AND XIA Y. 2012. Palladium nanocrystals enclosed by $\{100\}$ and $\{111\}$ facets in controlled proportions and their catalytic activities for formic acid oxidation. Energy Environ Sci 5: 6352-6357.

KALE MJ, AVANESIAN T AND CHRISTOPHER P. 2014. Direct Photocatalysis by Plasmonic Nanostructures. ACS Catalysis 4: 116-128.

KANG L, HAN X, CHU J, XIONG J, HE X, WANG HL AND XU P. 2015. In Situ Surface-Enhanced Raman Spectroscopy Study of Plasmon-Driven Catalytic Reactions of 4-Nitrothiophenol under a Controlled Atmosphere. Chem Cat Chem 7: 1004-1010.

KIM D, RESASCO J, YU Y, ASIRI AM AND YANG P. 2014. Synergistic geometric and electronic effects for electrochemical reduction of carbon dioxide using goldcopper bimetallic nanoparticles. Nature Commun 5: 4948.

KLAR T, PERNER M, GROSSE S, VON PLESSEN G, SPIRKL W AND FELDMANN J. 1998. Surface-Plasmon Resonances in Single Metallic Nanoparticles. Phys Rev Lett 80: 4249-4252.
KOGURE K AND HAMA S. 2015. Nanomaterials for Cosmetics. Encyclopedia of Polymeric Nanomaterials. In: Kobayashi S and Müllen K (Eds), Springer Berlin Heidelberg: Berlin, Heidelberg, p. 1349-1352.

LAMER VK AND DINEGAR RH. 1950. Theory, Production and Mechanism of Formation of Monodispersed Hydrosols. J Am Chem Soc 72: 4847-4854.

LEE K-S AND EL-SAYED MA. 2006. Gold and Silver Nanoparticles in Sensing and Imaging: Sensitivity of Plasmon Response to Size, Shape, and Metal Composition. J Phys Chem B 110: 19220-19225.

LEITE ER AND RIBEIRO C. 2012. Crystallization and Growth of Colloidal Nanocrystals. Springer, $14 \mathrm{p}$.

LEPAGE JF ET AL. 1997. Preparation of Solid Catalysts: Sections 2.0 and 2.1. In: Handbook of Heterogeneous Catalysis, Wiley-VCH Verlag GmbH, p. 49-138.

LIAO HG, JIANG YX, ZHOU ZY, CHEN SP AND SUN SG. 2008. Shape-Controlled Synthesis of Gold Nanoparticles in Deep Eutectic Solvents for Studies of StructureFunctionality Relationships in Electrocatalysis. Angew Chem 120: 9240-9243.

LINIC S, ASLAM U, BOERIGTER C AND MORABITO M. 2015. Photochemical transformations on plasmonic metal nanoparticles. Nat Mater 14: 567-576.

LINIC S, CHRISTOPHER P AND INGRAM DB. 2011. Plasmonic-metal nanostructures for efficient conversion of solar to chemical energy. Nat Mater 10: 911-921.

LOHSE SE, BURROWS ND, SCARABELLI L, LIZMARZÁN LM AND MURPHY CJ. 2014. Anisotropic Noble Metal Nanocrystal Growth: The Role of Halides. Chem Mater 26: 34-43.

LU X, AU L, MCLELLAN J, LI Z-Y, MARQUEZ M AND XIA Y. 2007. Fabrication of Cubic Nanocages and Nanoframes by Dealloying Au/Ag Alloy Nanoboxes with an Aqueous Etchant Based on $\mathrm{Fe}(\mathrm{NO} 3) 3$ or $\mathrm{NH} 4 \mathrm{OH}$. Nano Lett 7: 1764-1769.

LU X, RYCENGA M, SKRABALAK SE, WILEY B AND XIA Y. 2009. Chemical Synthesis of Novel Plasmonic Nanoparticles. Annu Rev Phys Chem 60: 167-192.

MÉTRAUX GS, CAO YC, RONGCHAO JIN AND MIRKIN CA. 2003. Triangular Nanoframes Made of Gold and Silver. Nano Lett 3: 519-522.

MIZUNO N AND MISONO M. 1998. Heterogeneous Catalysis. Chem Rev 98: 199-218.

NIKOOBAKHT B AND EL-SAYED MA. 2003. Preparation and Growth Mechanism of Gold Nanorods (NRs) Using Seed-Mediated Growth Method. Chem Mater 15: $1957-$ 1962.

NIU W AND LU X. 2015. Metallic Nanostructures: Fundamentals. Metallic Nanostructures: From Controlled Synthesis to Applications. In: Xiong Y and Lu X (Eds), Springer International Publishing: Cham, p. 1-47. 
NIU W, ZHANG L AND XU G. 2013. Seed-mediated growth of noble metal nanocrystals: crystal growth and shape control. Nanoscale 5: 3172-3181.

NORSKOV JK, BLIGAARD T, HVOLBAEK B, ABILD-PEDERSEN F, CHORKENDORFF I AND CHRISTENSEN CH. 2008. The nature of the active site in heterogeneous metal catalysis. Chem Soc Rev 37: 2163 2171.

NORSKOV JK, STUDT F, ABILD-PEDERSEN F AND BLIGAARD T. 2014. Heterogeneous Catalysis and a Sustainable Future. In: Fundamental Concepts in Heterogeneous Catalysis, J Wiley \& Sons, Inc, p. 1-5.

PANG B, YANG X AND XIA Y. 2016. Putting gold nanocages to work for optical imaging, controlled release and cancer theranostics. Nanomedicine 11: 1715-1728.

PARK J, JOO J, KWON SG, JANG Y AND HYEON T. 2007. Synthesis of monodisperse spherical nanocrystals. Angew Chem Int Ed 46: 4630-4660.

POLSHETTIWAR V AND VARMA RS. 2010. Green chemistry by nano-catalysis. Green Chem 12(5): 743-754.

PREVO BG, ESAKOFF SA, MIKHAILOVSKY A AND ZASADZINSKI JA. 2008. Scalable Routes to Gold Nanoshells with Tunable Sizes and Response to NearInfrared Pulsed-Laser Irradiation. Small 4: 1183-1195.

RODRIGUES T, DA SILVA AM, MACEDO A, FARINI B, ALVES R AND CAMARGO PC. 2015. Probing the catalytic activity of bimetallic versus trimetallic nanoshells. J Mater Sci 50: 5620-5629.

RODRIGUES TS, DA SILVA AGM, DE MOURA ABL, FREITAS IG AND CAMARGO PHC. 2016a. Rational design of plasmonic catalysts: matching the surface plasmon resonance with lamp emission spectra for improved performance in AgAu nanorings. RSC Adv 6: 62286-62290.

RODRIGUES TS, DA SILVA AGM, DE MOURA ABL, GEONMONOND RS AND CAMARGO PHC. 2017. $\mathrm{AgAu}$ Nanotubes: Investigating the Effect of Surface Morphologies and Optical Properties over Applications in Catalysis and Photocatalysis. J Braz Chem Soc 28: 16301638.

RODRIGUES TS, DA SILVA AHM, DA SILVA AGM, CEARA DG, GOMES JF, ASSAF JM AND CAMARGO PHC. 2016b. Hollow AgPt/SiO2 nanomaterials with controlled surface morphologies: is the number of Pt surface atoms imperative to optimize catalytic performances? Catal Sci Technol 6: 2162-2170.

SCHLÖGL R. 2015. Heterogeneous Catalysis. Angew Chem Int Ed 54: 3465-3520.

SHARMA N, OJHA H, BHARADWAJ A, PATHAK DP AND SHARMA RK. 2015. Preparation and catalytic applications of nanomaterials: a review. RSC Adv 5: 53381-53403.

STAMENKOVIC VR, FOWLER B, MUN BS, WANG G, ROSS PN, LUCAS CA AND MARKOVIĆ NM. 2007.
Improved Oxygen Reduction Activity on Pt3Ni(111) via Increased Surface Site Availability. Science 315: 493-497.

STILES PL, DIERINGER JA, SHAH NC AND VAN DUYNE RP. 2008. Surface-Enhanced Raman Spectroscopy. Annu Rev Anal Chem 1: 601-626.

SUN M AND XU H. 2012. A Novel Application of Plasmonics: Plasmon-Driven Surface-Catalyzed Reactions. Small 8: 2777-2786.

SUN Y, MAYERS B AND XIA Y. 2003. Metal Nanostructures with Hollow Interiors. Adv Mater 15: 641-646.

SUN Y AND XIA Y. 2002. Shape-Controlled Synthesis of Gold and Silver Nanoparticles. Science 298: 2176-2179.

THANH NTK, MACLEAN N AND MAHIDDINE S. 2014. Mechanisms of Nucleation and Growth of Nanoparticles in Solution. Chem Rev 114: 7610-7630.

VAJTAI R. 2013. Science and Engineering of Nanomaterials. Springer Handbook of Nanomaterials. In: Vajtai R (Ed), Springer: Berlin, Heidelberg, p. 1-36.

VISWANATHA R AND SARMA DD. 2007. Growth of Nanocrystals in Solution, In: Nanomaterials Chemistry: Recent Developments and New Directions, p. 139-160.

WALT DR. 2002. Nanomaterials: Top-to-bottom functional design. Nat Mater 1: 17-18.

WANG JL, ANDO RA AND CAMARGO PHC. 2014. Investigating the plasmon-mediated catalytic activity of agau nanoparticles as a function of composition: Are two metals better than one? ACS Catalysis 4: 3815-3819.

WANG Y, PENG HC, LIU J, HUANG CZ AND XIA Y. 2015. Use of Reduction Rate as a Quantitative Knob for Controlling the Twin Structure and Shape of Palladium Nanocrystals. Nano Lett 15: 1445-1450.

WELLS A AND MEYER HP. 2014. Biocatalysis as a Strategic Green Technology for the Chemical Industry. ChemCatChem 6: 918-920.

WILEY B, SUN Y AND XIA Y. 2007. Synthesis of silver nanostructures with controlled shapes and properties. Acc Chem Res 40: 1067-1076.

WILLETS KA AND VAN DUYNE RP. 2007. Localized Surface Plasmon Resonance Spectroscopy and Sensing. Annu Rev Anal Chem 58: 267-297.

XIA X, WANG Y, RUDITSKIY A AND XIA Y. 2013. $25^{\text {th }}$ Anniversary Article: Galvanic Replacement: A Simple and Versatile Route to Hollow Nanostructures with Tunable and Well-Controlled Properties. Adv Mater 25: 6313-6333.

XIA Y, GILROY KD, PENG HC AND XIA X. 2017. SeedMediated Growth of Colloidal Metal Nanocrystals. Angew Chem Int Ed 56: 60-95.

XIA Y, XIONG Y, LIM B AND SKRABALAK SE. 2009. Shape-Controlled Synthesis of Metal Nanocrystals: Simple Chemistry Meets Complex Physics? Angew Chem Int Ed 48: 60-103. 
XIONG Y AND LU X. 2014. Metallic Nanostructures: From Controlled Synthesis to Applications. In: Xiong Y and Lu $\mathrm{X}$ (Eds), Springer International Publishing, $301 \mathrm{p}$.

XIONG Y AND XIA Y. 2007. Shape-Controlled Synthesis of Metal Nanostructures: The Case of Palladium. Adv Mater 19: 3385-3391.

YIN Y, RIOUX RM, ERDONMEZ CK, HUGHES S, SOMORJAI GAAND ALIVISATOS AP. 2004. Formation of Hollow Nanocrystals Through the Nanoscale Kirkendall Effect. Science 304: 711-714.
YOU H AND FANG J. 2016. Particle-mediated nucleation and growth of solution-synthesized metal nanocrystals: A new story beyond the LaMer curve. Nanotoday 11: 145-167.

ZHANG Q, LI W, MORAN C, ZENG J, CHEN J, WEN LP AND XIA Y. 2010. Seed-Mediated Synthesis of Ag Nanocubes with Controllable Edge Lengths in the Range of 30-200 nm and Comparison of Their Optical Properties. J Am Chem Soc 132: 11372-11378. 